\title{
ASYMPTOTIC ANALYSIS BY THE SADDLE POINT METHOD OF THE ANICK-MITRA-SONDHI MODEL
}

\author{
DIEGO DOMINICI AND CHARLES KNESSL
}

Received 6 June 2003 and in revised form 12 December 2003

We consider a fluid queue where the input process consists of $N$ identical sources that turn on and off at exponential waiting times. The server works at the constant rate $c$ and an on source generates fluid at unit rate. This model was first formulated and analyzed by Anick et al. (1982). We obtain an alternate representation of the joint steady-state distribution of the buffer content and the number of on sources. This is given as a contour integral that we then analyze in the limit $N \rightarrow \infty$. We give detailed asymptotic results for the joint distribution as well as the associated marginal and conditional distributions. In particular, simple conditional limits laws are obtained. These show how the buffer content behaves conditioned on the number of active sources and vice versa. Numerical comparisons show that our asymptotic results are very accurate even for $N=20$.

\section{Introduction}

In traditional queueing theory, one usually considers a system with one or more servers, at which customers arrive to receive some kind of service. As all servers may be busy, the customers may have to wait for service in a queue. Since there is uncertainty about the actual arrival times of the customers and/or their service requirements, queues are typically modeled stochastically as random processes.

In the last twenty years, models have appeared in which a continuous quantity, referred to as a fluid, takes the role of the number of customers or queue length. In these models, fluid flows into a reservoir according to some stochastic process. The server may be viewed as a tap at the bottom of the reservoir allowing fluid to flow out. The rate at which this happens is often constant, but may also be stochastic. Since the fluid reservoir takes the role of the traditional customer queue, it is often referred to as a fluid queue. A third term which is often encountered is fluid buffer, stressing the fact that the storage of fluid is temporary to prevent loss of fluid at times when the input rate exceeds the output rate. Such models are used as approximations to discrete queuing models of manufacturing systems, high-speed data networks, transportation systems, in the theory of dams, and so forth. The distribution of the fluid level or buffer content, the average buffer content, 
the overflow probability (in the case of a finite buffer), and the output process are the main quantities of interest in these fluid models.

In the last decade, the literature on queueing theory has paid considerable attention to Markov-modulated fluid models [32, 41]. In these models, a fluid buffer is either filled or depleted, or both, at rates which are determined by the current state of a background Markov process, also called Markovian random environment.

1.1. The Markov-modulated fluid model. In this section, we describe a general model of fluid entering and leaving a single buffer system [26]. Let $X(t)$ denote the amount of fluid at time $t$ in the buffer. Furthermore, let $Z(t)$ be a continuous-time Markov process; $Z(t)$ will be said to evolve "in the background." We will assume that $Z(t)$ has a finite-state space $\mathcal{N}$ with $\mathcal{N}=\{0,1, \ldots, N\}$.

The buffer content $X(t)$ is regulated (or driven) by $Z(t)$ in such a way that the net input rate into the buffer (i.e., the rate of change of its content) is $\mathrm{d}[Z(t)]$. The function $\mathrm{d}(\cdot)$ is called the drift function. When the buffer capacity is infinite, the dynamics of $X(t)$ are given by

$$
\frac{d X}{d t}= \begin{cases}\mathrm{d}[Z(t)], & X(t)>0 \\ \max \{\mathrm{d}[Z(t)], 0\}, & X(t)=0\end{cases}
$$

The condition at $X(t)=0$ ensures that the process $X(t)$ does not become negative. When the buffer capacity is finite, say $K$, the dynamics are given by

$$
\frac{d X}{d t}= \begin{cases}\mathrm{d}[Z(t)], & 0<X(t)<K, \\ \max \{\mathrm{d}[Z(t)], 0\}, & X(t)=0, \\ \min \{\mathrm{d}[Z(t)], 0\}, & X(t)=K .\end{cases}
$$

The condition at $X(t)=K$ prevents the buffer content from exceeding $K$.

One of the main reasons why Markov-modulated fluid models have attracted so much attention is that they are relevant for modelling telecommunications networks. In the literature, more attention has been paid to models in which the buffer capacity is infinitely large because this case is easier to analyze. For most practical situations in telecommunications, the infinite buffer case is a good approximation for the finite buffer case since overflow of the buffer is assumed to be extremely rare. For highly loss-sensitive traffic, allowed loss fractions in the order of $10^{-6}$ to $10^{-10}$ are typical [9].

Unlike classical queueing models which assume renewal arrivals, Markov-modulated fluid models can capture autocorrelations in arrival processes. The continuous nature of the fluid also makes them more tractable analytically. Many results have been obtained for a variety of fluid queueing systems $[2,4,12,20,25,27,30,38,39,43,44,48]$.

In many applications, the process $Z(t)$ evolves as a finite-state birth-death process. Birth-death processes have wide applications in many practical problems [13]. They can be regarded as continuous time analogs of random walks. 
The paper which has become the main reference for birth-death fluid models was written by Anick et al. [3]. We will refer to their model as the Anick-Mitra-Sondhi (AMS) model. The AMS model describes an infinitely large fluid buffer which is fed by $N$ identical exponential on-off sources and emptied by an output channel with constant capacity c. An on source turns off at rate 1 and an off source turns on at rate $\lambda$. Thus, the net input is regulated by a specific birth-death process $Z(t)$ with state space $\mathcal{N}$, birth rate $\lambda_{k}=\lambda(N-k)$, death rate $\mu_{k}=k$, and drift function $\mathrm{d}(k)=k-c$. The rates are conditioned on $Z(t)=k, 0 \leq k \leq N$.

In a way, the AMS model was a generalization of the earlier model of Kosten $[21,22]$, where the limiting case $N \rightarrow \infty, \lambda \rightarrow 0$ (with $\lambda N$ fixed) is considered. According to the authors in [3], their model and its variants had also been proposed in other previous papers $[7,15,40]$.

In [23], Kosten generalizes the AMS model by considering the same problem for a Multi Group Finite Source (MGFS) system consisting of $m$ groups of identical sources and a single shared buffer. In [24], Kosten considers a central processor, working at uniform speed, that receives information at uniform rates from a number of sources and a buffer which stores the information that cannot be handled directly. He shows that the stationary probability of overflow $G(x)$ satisfies $G(x) \sim C e^{-\alpha x}$ as $x \rightarrow \infty$. For fairly complex aggregates of sources, a method is developed (called the decomposition method) to determine the exponent $\alpha$. The coefficient $C$ is determined by simulation.

Daigle and Langford [10] used the AMS model to study packet voice communication systems. Tucker [50] also used a similar model, but the server capacity was defined as an integer number of information units and the model had finite buffer size. Results on the buffer content distribution, cell loss and delay were given along with simulation comparisons.

The feature common to the above models is that they all assume a continuous time distribution for the on-off intervals. On the other hand, Li [28] introduced a discrete time model with a finite number of on-off sources and geometric distributions for the on-off intervals. He assumed that in one time unit, only one on-off source can change state. The channel capacity was assumed to be an integer number of sources and the buffer size could be either zero (burst switching-clipping case) or infinity (packet switching case).

In [33], Mitra shows how to deal with states $i \in \mathcal{N}$ for which $\mathrm{d}(i)=0$. He also generalizes the AMS model in two ways. Again, he considers a buffer which receives input from $N$ i.i.d. exponential sources, called producers. However, now the output is also assumed to be Markov-modulated by $M$ i.i.d. exponential consumers. A second generalization is that he considers the case of finite buffer capacity.

In the manufacturing literature, Wijngaard [55] has analyzed the model treated in [33] for the case of one producing and one consuming machine. Sevast'yanov [45] has given an innovative approximation for a production line model of many stages, which incorporates the exact solution of a pair of stages. All of this work assumes finite capacity buffers. Another extension of the AMS model is presented in [37], where the authors allow the possibility of two sources of input, one slow source of a fluid and another source that generates packets. 
Some birth-death fluid queues with finite $\mathcal{N}$ have also been proposed as approximative models when the modulating process in the original model is a multidimensional Markov chain, see [29]. In [8], the authors allow the modulating process to be an arbitrary birthdeath process on a finite-state space, but require the drift function $\mathrm{d}(i)$ to have a particular sign structure. An analysis without any restriction on the sign structure of the drift vector has been given in the survey paper [51].

Until recently, authors have mostly focused on stationary probabilities. The transient analysis of stochastic fluid models, that is, the analysis of the fluid distribution in the buffer at an arbitrary time $t$, is a complex and computationally intensive task. There are only a few papers dedicated to the transient behavior of fluid queues with Markov input. Narayanan and Kulkarni [35] derive explicit expressions for the Laplace transform of the joint distribution of the first time the buffer becomes empty and the state of the Markov process at that time.

The Laplace transform has been often used to evaluate the transient behavior of fluid flow models. In [19], Ren and Kobayashi studied the transient distribution of the buffer content for the AMS model. The same authors deal with the case of multiple types of inputs in [18]. These studies have been extended to the Markov-modulated input rate model by Tanaka et al. in [49].

Another approach to transient behavior is due to Sericola [42]. This leads to a numerically stable recursive method for computing transient and first passage distributions, and applies to general rate and drift functions.

The equilibrium probability that the buffer content exceeds $x, \operatorname{Pr}[X(\infty)>x]$, in the AMS model has been studied asymptotically, as $N \rightarrow \infty$, by Weiss [53] and Morrison [34]. The exact expression in [3] is convenient for calculating this probability for moderate values of $N$. However, when $N$ is large, it is useful to have asymptotic approximations.

Weiss [53] (see also [46]) analyzed the large $N$ asymptotics using the theory of large deviations. This yields an approximation of the form $\operatorname{Pr}[X(\infty)>N B] \approx \exp [-\mathrm{I}(B) N]$, where $\mathrm{I}(B)$ is characterized as a solution of a variational problem. Overflow asymptotics of this type for more general Markov-modulated fluid models are derived in [31]. Duffield [11] considers time-dependent asymptotics for the overflow probability.

Morrison [34] investigated the AMS model starting from the exact expression in [3], using the Euler-Maclaurin formula. He constructed different asymptotic approximations to $\operatorname{Pr}[X(\infty)>x]$ for the three scales $x=O(1 / N), x=O(1)$, and $x=O(N)$.

The heavy traffic analysis of the AMS model, where $N \rightarrow \infty$ and $c / N=\lambda /(\lambda+1)+$ $O\left(N^{-1 / 2}\right)$, was studied by Knessl and Morrison [16]. In this limit, computing the joint steady-state distribution of the buffer content and the number of on sources can be reduced to solving a partial differential equation, which is forward parabolic in a part of the domain and backward parabolic in the remaining portion.

In this paper, we analyze the joint distribution of the buffer content and number of on sources in the AMS model asymptotically, as the number of sources $N \rightarrow \infty$. Our results extend those obtained in [34].

In Section 2, we describe the AMS model in more detail and in Section 3, we derive an integral representation of the solution from which the spectral representation obtained by the authors in [3] can be easily derived. In Sections 4, 5, 6, 7, 8, and 9, we obtain 
asymptotic expansions from the exact solution by using the saddle point method. We have to consider eleven relevant regions of the two-dimensional state space. In Section 10, we summarize our results and numerically compare our approximations with the exact solution. In Section 11, we analyze the conditional and marginal distributions.

\section{The Anick-Mitra-Sondhi model}

In this model, a data-handling switch receives messages from $N$ mutually independent sources, which independently and asynchronously alternate between on and off states. The on and off periods are exponentially distributed for each source. These two distributions, while not necessarily identical, are common to all sources.

The unit of time is selected to be the average on period and the average off period is denoted by $1 / \lambda$. Thus, if we denote by On (Off) the random variables measuring the elapsed time when a source is in the on (off) state, we have

$$
\begin{gathered}
\operatorname{Pr}[\text { On } \leq T]=\int_{0}^{T} e^{-s} d s, \quad \operatorname{Pr}[\text { Off } \leq T]=\int_{0}^{T} \lambda e^{-\lambda s} d s, \\
\mathrm{E}[\operatorname{Pr}(\text { a source is on })]=\frac{\lambda}{\lambda+1} .
\end{gathered}
$$

An on source will generate packets of information at a uniform rate that we assume, without loss of generality, to be 1 packet per unit of time. While a source is off, it generates no packets. Thus, when $k$ sources are on simultaneously, the instantaneous receiving rate at the switch is $k$. The switch stores or buffers the incoming information that is in excess of the maximum transmission rate $c$ of an output channel (thus, $c$ is also the ratio of the output channel capacity to an on source's transmission rate). We assume that $c$ is not an integer.

Let $Z(t)$ be the number of on sources at time $t$, and let $X(t)$ be the buffer content. As long as the buffer is not empty, the instantaneous rate of change of the buffer content is $Z(t)-c$. Once the buffer is empty, it remains so as long as $Z(t)<c$. We assume that the buffer capacity is infinite, so we need the following stability condition:

$$
\frac{\lambda}{\lambda+1}<\gamma<1, \quad \gamma=\frac{c}{N}
$$

to ensure the existence of a steady-state distribution. Note that $c<N$ implies that the buffer may be nonempty and $c>(\lambda /(\lambda+1)) N$ means that the output rate exceeds the average input rate.

Here, we consider only the equilibrium probabilities defined by

$$
F_{k}(x)=\lim _{t \rightarrow \infty} \operatorname{Pr}[Z(t)=k, X(t) \leq x], \quad 0 \leq k \leq N, x>0 .
$$

The balance equations are [3]

$$
(k-c) \frac{\partial F_{k}}{\partial x}=\lambda[N-(k-1)] F_{k-1}+(k+1) F_{k+1}-[\lambda(N-k)+k] F_{k}, \quad 0 \leq k \leq N,
$$


with

$$
F_{k}(x) \equiv 0, \quad k \notin[0, N]
$$

Moreover, if the number of on sources at any time exceeds $c$, then the buffer content increases and the buffer cannot stay empty. Hence,

$$
F_{k}(0)=0, \quad\lfloor c\rfloor+1 \leq k \leq N .
$$

Also,

$$
F_{k}(\infty)=\frac{1}{(1+\lambda)^{N}}\left(\begin{array}{c}
N \\
k
\end{array}\right) \lambda^{k}, \quad 0 \leq k \leq N,
$$

since $F_{k}(\infty)$ is the probability that $k$ out of $N$ sources are on simultaneously, and from (2.2), this follows a binomial distribution with parameter $\lambda /(\lambda+1)$.

In [3], Anick et al. derived the spectral representation of the solution to (2.5), (2.6), (2.7), and (2.8). They wrote (2.5) in matrix form:

$$
\operatorname{DF}^{\prime}(x)=\operatorname{MF}(x), \quad x \geq 0,
$$

where

$$
\mathbf{F}(x)=\left[F_{0}(x), F_{1}(x), \ldots, F_{N}(x)\right]^{T},
$$

D is an $(N+1) \times(N+1)$ diagonal matrix with entries

$$
\mathbf{D}_{i j}=(j-c) \delta_{i j}, \quad 0 \leq i, j \leq N,
$$

and $\mathbf{M}$ is an $(N+1) \times(N+1)$ tridiagonal matrix with entries

$$
\mathbf{M}_{i j}= \begin{cases}j, & j=i+1,0 \leq i \leq N-1, \\ -[\lambda(N-j)+j], & j=i, 0 \leq i \leq N, \\ \lambda(N-j), & j=i-1,1 \leq i \leq N, \\ 0, & |i-j| \geq 2 .\end{cases}
$$

Using (2.9), they represented the solution $\mathbf{F}(x)$ as

$$
\mathbf{F}(x)=\mathbf{F}(\infty)+\sum_{i=0}^{N-\lfloor c\rfloor-1} a_{i} e^{z_{i} x} \phi_{i}
$$

where $z_{i}$ is a negative eigenvalue and $\phi_{i}$ is the associated right eigenvector of the matrix $\mathbf{D}^{-1} \mathbf{M}$ :

$$
\mathbf{D}^{-1} \mathbf{M} \phi_{i}=z_{i} \phi_{i} .
$$


They showed that (2.5) and (2.7) imply conditions on the derivatives of $F_{N}(x)$ at $x=0$ :

$$
\frac{d^{n} F_{N}}{d x^{n}}(0)=0, \quad 0 \leq n \leq N-\lfloor c\rfloor-1
$$

From (2.15), they found the coefficients $a_{i}$ in (2.13) with the help of Vandermonde determinants. Asymptotic and numerical results for the overflow probability of a predefined buffer backlog were also presented.

\section{Exact solution}

Here we will solve the problem (2.5), (2.6), (2.7), and (2.8) by a different method than that used in [3]. This will lead to an alternate form of the solution that will prove useful for asymptotic analysis.

Looking for a solution to (2.5) of the form

$$
F_{k}(x)=e^{\theta x} h_{k}(\theta)
$$

we get the following difference equation for $h_{k}(\theta)$ :

$$
\lambda(N-k+1) h_{k-1}+(k+1) h_{k+1}-[\lambda(N-k)+k+(k-c) \theta] h_{k}=0, \quad 0 \leq k \leq N .
$$

To solve (3.2), we represent $h_{k}(\theta)$ as an integral:

$$
h_{k}(\theta)=\frac{1}{2 \pi i} \oint_{\mathscr{C}} \frac{H(\theta, w)}{w^{N-k+1}} d w
$$

where $\mathscr{C}$ is a small loop around the origin in the complex plane and $H(\theta, w)$ is an analytic function of $w$ inside $\mathscr{C}$. Using (3.3) in (3.2) and integrating by parts gives an ODE for $H(\theta, w)$

$$
\frac{\partial H}{\partial w}=\frac{(w+\gamma \theta-1-\theta) N}{w^{2}+(\lambda-\theta-1) w-\lambda} H
$$

whose solution is

$$
H(\theta, w)=\left[1-R_{1}(\theta) w\right]^{N V(\theta)}\left[1+R_{2}(\theta) w\right]^{N[1-V(\theta)]}
$$

with

$$
\begin{array}{ll}
V(\theta)=\frac{1}{2}\left[1+\frac{(2 \gamma-1) \theta-\lambda-1}{\Delta(\theta)}\right], & \Delta(\theta)=\sqrt{(\theta+1-\lambda)^{2}+4 \lambda} \\
R_{1}(\theta)=\frac{1}{2 \lambda}[\Delta(\theta)+(\lambda-1-\theta)], & R_{2}(\theta)=\frac{1}{2 \lambda}[\Delta(\theta)-(\lambda-1-\theta)] .
\end{array}
$$


Using the binomial theorem, we can also represent $h_{k}(\theta)$ as

$$
h_{k}(\theta)=\sum_{i=0}^{N-k}\left(\begin{array}{c}
N V(\theta) \\
i
\end{array}\right)\left(\begin{array}{c}
N[1-V(\theta)] \\
N-k-i
\end{array}\right)\left[-R_{1}(\theta)\right]^{i}\left[R_{2}(\theta)\right]^{N-k-i}
$$

which shows that $h_{k}(\theta)$ is a polynomial in $\theta$ of degree $N-k$ :

$$
\begin{aligned}
h_{N}(\theta) & =1, \\
h_{N-1}(\theta) & =\frac{1+(1-\gamma) \theta}{\lambda} N, \\
h_{N-2}(\theta) & =\frac{(\gamma-1) \theta^{2}+(\phi-2) \theta-1}{2 \lambda^{2}} N+\frac{[1+(1-\gamma) \theta]^{2}}{2 \lambda^{2}} N^{2}, \\
& \vdots \\
h_{N-k}(\theta) & =c_{1}(\theta) N+\cdots+c_{k-1}(\theta) N^{k-1}+\frac{[1+(1-\gamma) \theta]^{k}}{\lambda^{k} k !} N^{k}, \quad 1 \leq k \leq N .
\end{aligned}
$$

The polynomials $h_{k}(\theta)$ are related to the Krawtchouk polynomials [47]. The functions $c_{k}(\theta)$ are entire (polynomial) functions of $\theta$, that may be computed from (3.8).

Since $H$ is analytic inside $\mathscr{C}$, we have

$$
h_{N+1}(\theta)=\frac{1}{2 \pi i} \oint_{\mathscr{C}} H(\theta, w) d w=0
$$

Imposing the boundary condition (2.6) yields $h_{k}(\theta)=0$ for $k \leq-1$. Thus,

$$
\frac{\partial^{k} H}{\partial w^{k}}(\theta, 0)=0, \quad k \geq N+1
$$

and therefore $H(\theta, w)$ must be a polynomial in $w$ of degree $\leq N$. In view of (3.5), we must have

$$
V\left(\theta_{j}\right)=\frac{j}{N}, \quad 0 \leq j \leq N,
$$

which gives the equation for the eigenvalues $\theta_{j}$. Since from (2.8) we need $F_{k}(x) \rightarrow F_{k}(\infty)$ for $x \rightarrow \infty$, we consider only the negative eigenvalues. Solving for $\theta_{j}$ in (3.12), we get

$$
\theta_{j}=-\sigma\left(\frac{j}{N}\right)<0, \quad 0 \leq j \leq N-\lfloor c\rfloor-1,
$$

where

$$
\begin{gathered}
\sigma(x)=\frac{\rho+2(\lambda-1)(1-x) x+(1-2 x) \sqrt{\rho^{2}+4 \lambda(1-x) x}}{2(\gamma-x)(1-\gamma-x)}, \\
\rho=\gamma-\lambda+\lambda \gamma
\end{gathered}
$$


with $0<\rho<1$ from (2.3). Combining the above results and using (2.8), we find the spectral representation of the solution

$$
F_{k}(x)=\frac{1}{(1+\lambda)^{N}}\left(\begin{array}{l}
N \\
k
\end{array}\right) \lambda^{k}+\sum_{j=0}^{N-\lfloor c\rfloor-1} a_{j} e^{\theta_{j} x} h_{k}\left(\theta_{j}\right)
$$

where the constants $a_{j}$ are to be determined. To find $a_{j}$, we employ arguments different from those in [3].

Taking the Laplace transform

$$
\mathscr{F}_{k}(\vartheta)=\int_{0}^{\infty} e^{-\vartheta x} F_{k}(x) d x
$$

in (2.5) and using the boundary conditions (2.7) for $k \geq\lfloor c\rfloor+1$ gives

$$
(k-c) \vartheta \mathscr{F}_{k}=\lambda[N-(k-1)] \mathscr{F}_{k-1}+(k+1) \mathscr{F}_{k+1}-[\lambda(N-k)+k] \mathscr{F}_{k}, \quad\lfloor c\rfloor+1 \leq k \leq N .
$$

Equation (3.18) has two independent solutions $h_{k}(\vartheta)$ and $h_{k}^{*}(\vartheta)$, with $h_{k}(\vartheta)$ given by $(3.3)$ and

$$
h_{k}^{*}(\vartheta)=\frac{1}{2 \pi i} \oint_{\mathscr{C}} H^{*}(\vartheta, w) \frac{1}{w^{k+1}} d w
$$

where $\mathscr{C}$ is a small loop around the origin and

$$
H^{*}(\vartheta, w)=\left[1-\lambda R_{2}(\vartheta) w\right]^{N V(\theta)}\left[1+\lambda R_{1}(\vartheta) w\right]^{N[1-V(\vartheta)]} .
$$

Using the binomial theorem, we get from (3.19)

$$
h_{k}^{*}(\vartheta)=\lambda^{k} \sum_{i=0}^{k}\left(\begin{array}{c}
N V(\vartheta) \\
i
\end{array}\right)\left(\begin{array}{c}
N[1-V(\vartheta)] \\
k-i
\end{array}\right)\left[-R_{2}(\vartheta)\right]^{i}\left[R_{1}(\vartheta)\right]^{k-i}
$$

From (3.21), we have

$$
\begin{aligned}
h_{0}^{*}(\vartheta) & =1, \\
h_{1}^{*}(\vartheta) & =(\lambda-\gamma \vartheta) N, \\
h_{2}^{*}(\vartheta) & =\frac{1}{2}\left[-\lambda^{2}+(\gamma \lambda+\lambda-\gamma) \vartheta-\gamma \vartheta^{2}\right] N+\frac{(\lambda-\gamma \vartheta)^{2}}{2} N^{2}, \\
& \vdots \\
h_{k}^{*}(\vartheta) & =c_{1}^{*}(\vartheta) N+\cdots+c_{k-1}^{*}(\vartheta) N^{k-1}+\frac{(\lambda-\gamma \vartheta)^{k}}{k !} N^{k}, \quad 1 \leq k \leq N,
\end{aligned}
$$


where the $c_{k}^{*}(\vartheta)$ are polynomials that can be identified from (3.21) using (3.6) and (3.7). Therefore, the general solution to (3.18) is given by

$$
\mathscr{F}_{k}(9)=A_{1}(9) h_{k}(\vartheta)+A_{2}(\vartheta) h_{k}^{*}(\vartheta), \quad\lfloor c\rfloor+1 \leq k \leq N,
$$

with $A_{1}(\vartheta), A_{2}(\vartheta)$ still undetermined. We note that $h_{k}$ and $h_{k}^{*}$ are entire functions of $\vartheta$. Since $h_{N+1}(9)=0$ but $h_{N+1}^{*}(\vartheta) \neq 0$, we see from (2.6) that we need $A_{2}(\vartheta)=0$ so that

$$
\mathscr{F}_{k}(\vartheta)=A_{1}(\vartheta) h_{k}(\vartheta) .
$$

Taking the Laplace transform in (3.16) yields

$$
\mathscr{F}_{k}(\vartheta)=\frac{1}{(1+\lambda)^{N}}\left(\begin{array}{l}
N \\
k
\end{array}\right) \lambda^{k} \frac{1}{\vartheta}+\sum_{j=0}^{N-|c|-1} a_{j} h_{k}\left(\theta_{j}\right) \frac{1}{\vartheta-\theta_{j}},
$$

from which we see that the only singularities of $\mathscr{F}_{k}(\vartheta)$ are simple poles at $\vartheta=0$ and $\vartheta=\theta_{j}$, $0 \leq j \leq N-\lfloor c\rfloor-1$. Since $h_{k}(9)$ is entire, $A_{1}(9)$ must have poles at these points also. We thus rewrite (3.24) as

$$
\mathscr{F}_{k}(\vartheta)=B(\vartheta) \frac{1}{\vartheta}\left[\prod_{j=0}^{N-\lfloor c \mid-1} \frac{1}{\theta_{j}-\vartheta}\right] h_{k}(\vartheta),
$$

where $B(\vartheta)$ is an entire function. Using the inversion formula, we can represent $F_{k}(x)$ in the form

$$
F_{k}(x)=\frac{1}{2 \pi i} \int_{\mathrm{Br}^{+}} e^{x \vartheta} B(\vartheta) \frac{1}{\vartheta}\left[\prod_{j=0}^{N-\lfloor c\rfloor-1} \frac{1}{\theta_{j}-\vartheta}\right] h_{k}(\vartheta) d \vartheta,
$$

where $\mathrm{Br}^{+}$is a vertical contour on which $\operatorname{Re}(9)>0$. From (3.25), the residue of $\mathscr{F}_{k}(9)$ at $\vartheta=0$ is equal to

$$
\frac{1}{(1+\lambda)^{N}}\left(\begin{array}{l}
N \\
k
\end{array}\right) \lambda^{k}
$$

and from (3.5), we know that

$$
H(0, w)=\left(1+\frac{w}{\lambda}\right)^{N},
$$

which implies that

$$
h_{k}(0)=\left(\begin{array}{c}
N \\
k
\end{array}\right) \lambda^{k-N} .
$$


Thus, from (3.27), we get

$$
B(0)=\left(\frac{\lambda}{\lambda+1}\right)^{N} \prod_{j=0}^{N-\lfloor c\rfloor-1} \theta_{j}
$$

We determine $B(\vartheta)$ by examining the limit $\vartheta \rightarrow \infty$, which corresponds to considering the boundary condition at $x=0$.

From (3.6) and (3.7), we can easily obtain the asymptotic expressions

$$
\begin{aligned}
V(\vartheta) & \sim \gamma-\phi \frac{1}{\vartheta}, \quad \vartheta \longrightarrow \infty \\
R_{1}(\vartheta) & \sim \frac{1}{\vartheta}+(\lambda-1) \frac{1}{\vartheta^{2}}, \quad \vartheta \longrightarrow \infty \\
R_{2}(\vartheta) & \sim \frac{1}{\lambda} \vartheta+\frac{1-\lambda}{\lambda}+\frac{1}{\vartheta}, \quad \vartheta \longrightarrow \infty .
\end{aligned}
$$

Setting $w=u / \vartheta$ in (3.3) and using (3.32) yields

$$
\begin{aligned}
h_{k}(\vartheta) & \sim \vartheta^{N-k} \frac{1}{2 \pi i} \oint_{\mathscr{C}}\left(1+\frac{u}{\lambda}\right)^{(1-\gamma) N} \frac{d u}{u^{N-k+1}} \\
& =\left(\frac{\vartheta}{\lambda}\right)^{N-k}\left(\begin{array}{c}
(1-\gamma) N \\
N-k
\end{array}\right), \quad \vartheta \longrightarrow \infty .
\end{aligned}
$$

We also have

$$
\prod_{j=0}^{N-\lfloor c\rfloor-1} \frac{1}{\theta_{j}-\vartheta} \sim \frac{(-1)^{N-\lfloor c\rfloor}}{\vartheta^{N-\lfloor c\rfloor}}, \quad \vartheta \longrightarrow \infty
$$

Combining (3.33) and (3.34), we get

$$
\mathscr{F}_{k}(\vartheta) \sim B(\vartheta) \frac{(-1)^{N-\lfloor c\rfloor}}{\lambda^{N-k}}\left(\begin{array}{c}
(1-\gamma) N \\
N-k
\end{array}\right) \vartheta^{\lfloor c\rfloor-k-1}, \quad \vartheta \longrightarrow \infty
$$

Since (2.7) implies that

$$
\lim _{\vartheta \rightarrow \infty}\left[\vartheta \mathscr{F}_{k}(\vartheta)\right]=0,\lfloor c\rfloor+1 \leq k \leq N,
$$

we obtain

$$
B(\vartheta)=o\left(\vartheta^{k-\lfloor c\rfloor}\right), \quad \vartheta \longrightarrow \infty,\lfloor c\rfloor+1 \leq k \leq N
$$


Setting $k=\lfloor c\rfloor+1$, we see that $B(\vartheta)$ is an entire function that is $o(\vartheta)$ as $\vartheta \rightarrow \infty$. By the generalized Liouville theorem [17], $B(\vartheta)$ must be a constant

$$
B(\vartheta)=B(0)=\left(\frac{\lambda}{\lambda+1}\right)^{N} \prod_{j=0}^{N-\lfloor c\rfloor-1} \theta_{j}
$$

Therefore, we can write

$$
F_{k}(x)=\left(\frac{\lambda}{\lambda+1}\right)^{N} \frac{1}{2 \pi i} \int_{\mathrm{Br}^{+}} e^{x \theta} \frac{1}{\vartheta}\left[\prod_{j=0}^{N-\lfloor c\rfloor-1} \frac{\theta_{j}}{\theta_{j}-\vartheta}\right] h_{k}(\vartheta) d \vartheta
$$

Closing $\mathrm{Br}^{+}$in the half-plane $\operatorname{Re}(\vartheta) \leq 0$, we recover the spectral representation

$$
F_{k}(x)=\frac{1}{(1+\lambda)^{N}}\left(\begin{array}{l}
N \\
k
\end{array}\right) \lambda^{k}+\sum_{j=0}^{N-\lfloor c\rfloor-1} a_{j} e^{\theta_{j} x} h_{k}\left(\theta_{j}\right)
$$

with

$$
a_{j}=-\left(\frac{\lambda}{\lambda+1}\right)^{N} \prod_{\substack{i=0 \\ i \neq j}}^{N-\lfloor c\rfloor-1} \frac{\theta_{i}}{\theta_{i}-\theta_{j}}
$$

In conclusion, we have proved the following theorem.

Theorem 3.1. The solution of (2.5), (2.6), (2.7), and (2.8) is given by

$$
F_{k}(x)=\left(\frac{\lambda}{\lambda+1}\right)^{N} \frac{1}{2 \pi i} \int_{\mathrm{Br}^{+}} e^{x \vartheta} \frac{1}{\vartheta}\left[\prod_{j=0}^{N-\lfloor c\rfloor-1} \frac{\theta_{j}}{\theta_{j}-\vartheta}\right] h_{k}(\vartheta) d \vartheta
$$

where $\mathrm{Br}^{+}$is a vertical contour on which $\operatorname{Re}(9)>0$,

$$
h_{k}(\vartheta)=\frac{1}{2 \pi i} \oint_{\mathscr{C}} \frac{H(\vartheta, w)}{w^{N-k+1}} d w,
$$

$\mathscr{C}$ is a small loop around the origin in the complex $w$-plane

$$
\begin{gathered}
H(\vartheta, w)=\left[1-R_{1}(\vartheta) w\right]^{N V(\vartheta)}\left[1+R_{2}(\vartheta) w\right]^{N[1-V(\vartheta)]}, \\
V(\vartheta)=\frac{1}{2}\left[1+\frac{(2 \gamma-1) \vartheta-\lambda-1}{\Delta(\vartheta)}\right], \quad \Delta(\vartheta)=\sqrt{(\vartheta+1-\lambda)^{2}+4 \lambda}, \\
R_{1}(\vartheta)=\frac{1}{2 \lambda}[\Delta(\vartheta)+(\lambda-1-\vartheta)], \quad R_{2}(\vartheta)=\frac{1}{2 \lambda}[\Delta(\vartheta)-(\lambda-1-\vartheta)], \\
\theta_{j}=-\sigma\left(\frac{j}{N}\right)<0, \quad 0 \leq j \leq N-\lfloor c\rfloor-1, \\
\sigma(x)=\frac{\rho+2(\lambda-1)(1-x) x+(1-2 x) \sqrt{\rho^{2}+4 \lambda(1-x) x}}{2(\gamma-x)(1-\gamma-x)} .
\end{gathered}
$$


We have thus rederived, using different arguments, the spectral representation

$$
F_{k}(x)=\frac{1}{(1+\lambda)^{N}}\left(\begin{array}{l}
N \\
k
\end{array}\right) \lambda^{k}-\left(\frac{\lambda}{\lambda+1}\right)^{N} \sum_{j=0}^{N-\lfloor c\rfloor-1}\left[\prod_{\substack{i=0 \\
i \neq j}}^{N-\lfloor c\rfloor-1} \frac{\theta_{i}}{\theta_{i}-\theta_{j}}\right] e^{\theta_{j} x} h_{k}\left(\theta_{j}\right)
$$

obtained by the authors in [3]. We also derived the integral representation (3.42) that will prove more useful than (3.45) for asymptotic analysis. We note that the coefficients in the sum in (3.45) alternate in sign and this makes it very difficult to obtain asymptotic results for $F_{k}(x)$ from the spectral representation.

We study asymptotic properties of $F_{k}(x)$ as $N \rightarrow \infty$ for various ranges of $k$ and $x$. We will use (3.42), approximate the integrand for $N$ large, and then evaluate the integral over $\mathrm{Br}^{+}$asymptotically. We will introduce the scaled variables $(y, z)=(x / N, k / N)$ and consider the limit $N \rightarrow \infty$, with $y$ and $z$ fixed. In Section 4 , we will derive asymptotic approximations to $F_{k}(x)$ that are valid in most of the strip $\mathfrak{D}=\{(y, z): y \geq 0,0 \leq z \leq 1\}$, with the exception of a few boundary regions and one curve in $\mathfrak{D}$ (the interior of $\mathfrak{D}$ ). These other ranges are considered in Sections 5, 6, 7, 8, and 9.

\section{The main approximation}

We first evaluate the product in (3.42) for $N \rightarrow \infty$.

LeMma 4.1. Let

$$
P(\vartheta)=\prod_{j=0}^{N-\lfloor c\rfloor-1} \frac{\theta_{j}}{\theta_{j}-\vartheta}
$$

Then, for $N \rightarrow \infty$ and a fixed $\vartheta>\theta_{0}$, we have

$$
P(\vartheta) \sim \widetilde{P}(\vartheta ; N) \equiv \sqrt{\frac{-\theta_{0}}{\vartheta-\theta_{0}}} \exp [N \mu(\vartheta)]
$$

with

$$
\begin{gathered}
\mu(\vartheta)=-\frac{1}{2} \ln [\gamma(1-\gamma) \vartheta+\rho] \\
+\frac{\vartheta(1-2 \gamma)+\lambda+1}{2 \Delta(\vartheta)} \ln \left[\frac{[\gamma(1-\gamma) \vartheta+\rho][\lambda-1-\vartheta+\Delta(\vartheta)]}{(\lambda-1-\vartheta) \rho+(\lambda+1)^{2} \gamma(1-\gamma)+(1-\lambda) \gamma(1-\gamma) \vartheta+\Delta(\vartheta) \delta}\right], \\
\theta_{0}=-\frac{\rho}{\gamma(1-\gamma)}<0, \quad \delta=(1-\gamma)^{2} \lambda+\gamma^{2}>0 \\
\rho=\gamma \lambda+\gamma-\lambda .
\end{gathered}
$$

We note from (4.3) that $\mu(0)=0$. 
Proof. Since we assumed that $c$ is not an integer, we introduce the fractional part of $c$ defined by

$$
\alpha=c-\lfloor c\rfloor, \quad 0<\alpha<1
$$

which allows us to write $N-\lfloor c\rfloor$ as $(1-\gamma) N+\alpha$. Since

$$
\ln [P(\vartheta)]=\ln \left[\prod_{k=0}^{(1-\gamma) N+\alpha-1} \frac{\sigma(k / N)}{\vartheta+\sigma(k / N)}\right]=\sum_{k=0}^{(1-\gamma) N+\alpha-1} \ln \left[\frac{\sigma(k / N)}{\vartheta+\sigma(k / N)}\right],
$$

we can apply the Euler-Maclaurin formula [5] to get

$$
\begin{aligned}
\ln [P(\vartheta)]= & \frac{1}{2}\left\{\ln \left[\frac{\sigma(0)}{\vartheta+\sigma(0)}\right]+\ln \left[\frac{\sigma(1-\gamma+(\alpha-1) / N)}{\vartheta+\sigma(1-\gamma+(\alpha-1) / N)}\right]\right\} \\
& +\int_{0}^{(1-\gamma) N+\alpha-1} \ln \left[\frac{\sigma(k / N)}{\vartheta+\sigma(k / N)}\right] d k+o(1) .
\end{aligned}
$$

Changing variables from $k$ to $x=k / N$, we obtain

$$
\int_{0}^{(1-\gamma) N+\alpha-1} \ln \left[\frac{\sigma(k / N)}{\vartheta+\sigma(k / N)}\right] d k=\frac{1}{\varepsilon} \int_{0}^{1-\gamma-(1-\alpha) \varepsilon} \ln \left[\frac{\sigma(x)}{\vartheta+\sigma(x)}\right] d x
$$

with

$$
\varepsilon=\frac{1}{N}
$$

If we denote the indefinite integral in (4.8) by

$$
\mathrm{I}_{1}(z)=\int^{z} \ln \left[\frac{\sigma(x)}{\vartheta+\sigma(x)}\right] d x
$$

then we have, as $\varepsilon \rightarrow 0^{+}$,

$$
\int_{0}^{1-\gamma-(1-\alpha) \varepsilon} \ln \left[\frac{\sigma(x)}{\vartheta+\sigma(x)}\right] d x \sim \mathrm{I}_{1}\left[(1-\gamma)^{-}\right]-(1-\alpha) \mathrm{I}_{1}^{\prime}\left[(1-\gamma)^{-}\right] \varepsilon-\mathrm{I}_{1}(0)
$$

where $\mathrm{I}_{1}\left[(1-\gamma)^{-}\right]$means $\lim _{z \uparrow 1-\gamma} \mathrm{I}_{1}(z)$. From (3.14) we get

$$
\sigma(0)=\frac{\rho}{\gamma(1-\gamma)}, \quad \sigma(1-\gamma-\varepsilon) \sim \frac{\phi}{\varepsilon}, \quad \varepsilon \longrightarrow 0^{+}
$$

with

$$
\phi=\gamma+\lambda-\gamma \lambda
$$


Therefore,

$$
\mathrm{I}_{1}^{\prime}(1-\gamma)=\lim _{x \uparrow 1-\gamma} \ln \left[\frac{\sigma(x)}{\vartheta+\sigma(x)}\right]=\ln (1)=0 .
$$

To find $\mathrm{I}_{1}(z)$, we use integration by parts:

$$
\mathrm{I}_{1}(z)=-\int^{z} \ln \left[\frac{\vartheta}{\sigma(x)}+1\right] d x=-z \ln \left[\frac{\vartheta}{\sigma(z)}+1\right]-\vartheta \int^{z} x \frac{\sigma^{\prime}(x)}{[\vartheta+\sigma(x)] \sigma(x)} d x
$$

Since (3.12) implies that $V[-\sigma(x)]=x$, we can write (4.15) as

$$
\mathrm{I}_{1}(z)=-z \ln \left[\frac{\vartheta}{\sigma(z)}+1\right]-\vartheta \int^{z} \frac{V[-\sigma(x)]}{[\vartheta+\sigma(x)] \sigma(x)} \sigma^{\prime}(x) d x
$$

or, changing the integrating variable from $x$ to $\sigma$,

$$
\begin{aligned}
\mathrm{I}_{1}(z)= & -z \ln \left[\frac{\vartheta}{\sigma(z)}+1\right]-\vartheta \int^{\sigma(z)} \frac{V(-\sigma)}{(\vartheta+\sigma) \sigma} d \sigma \\
= & -z \ln \left[\frac{\vartheta}{\sigma(z)}+1\right]-\vartheta \int^{\sigma(z)} \frac{1}{2}\left[1-\frac{(2 \gamma-1) \sigma+\lambda+1}{\sqrt{(-\sigma+1-\lambda)^{2}+4 \lambda}}\right] \frac{1}{(\vartheta+\sigma) \sigma} d \sigma \\
= & -z \ln \left[\frac{\vartheta}{\sigma(z)}+1\right]-\frac{\vartheta}{2} \int^{\sigma(z)} \frac{1}{(\vartheta+\sigma) \sigma} d \sigma \\
& +\frac{\vartheta}{2} \int^{\sigma(z)} \frac{(2 \gamma-1) \sigma+\lambda+1}{\sqrt{(-\sigma+1-\lambda)^{2}+4 \lambda}} \frac{1}{(\vartheta+\sigma) \sigma} d \sigma .
\end{aligned}
$$

The first integral in (4.17) is

$$
\int \frac{1}{(\vartheta+\sigma) \sigma} d \sigma=\frac{1}{\vartheta} \ln \left[\frac{\sigma}{\sigma+\vartheta}\right]
$$

The second integral in (4.17) is quite complicated and after some calculation we find that

$$
\begin{aligned}
& \int \frac{(2 \gamma-1) \sigma+\lambda+1}{\sqrt{(-\sigma+1-\lambda)^{2}+4 \lambda}} \frac{1}{(\vartheta+\sigma) \sigma} d \sigma \\
& =\frac{1}{2 \vartheta} \ln \left\{\frac{\left[(\lambda+1)^{2}+(\lambda-1) \sigma-(\lambda+1) \Delta(-\sigma)\right]^{2}}{4 \lambda \sigma^{2}}\right\} \\
& \quad+\frac{\vartheta(1-2 \gamma)+\lambda+1}{\vartheta \Delta(\vartheta)} \ln \left[2 \frac{(\lambda-1-\vartheta) \sigma+(\lambda+1)^{2}+(1-\lambda) \vartheta+\Delta(\vartheta) \Delta(-\sigma)}{\vartheta+\sigma}\right] .
\end{aligned}
$$


Hence,

$$
\mathrm{I}_{1}(z)=\left(z-\frac{1}{2}\right) \ln \left[\frac{\sigma(z)}{9+\sigma(z)}\right]+Q_{1}[\sigma(z)]
$$

with

$$
\begin{aligned}
Q_{1}(\sigma)= & \frac{1}{4} \ln \left\{\frac{\left[(\lambda+1)^{2}+(\lambda-1) \sigma-(\lambda+1) \Delta(-\sigma)\right]^{2}}{4 \lambda \sigma^{2}}\right\} \\
& +\frac{\vartheta(1-2 \gamma)+\lambda+1}{2 \Delta(\vartheta)} \ln \left[2 \frac{(\lambda-1-\vartheta) \sigma+(\lambda+1)^{2}+(1-\lambda) \vartheta+\Delta(\vartheta) \Delta(-\sigma)}{\vartheta+\sigma}\right] .
\end{aligned}
$$

As $z \uparrow 1-\gamma$, we have

$$
Q_{1}[\sigma(z)] \sim-\frac{1}{4} \ln (\lambda)+\frac{\vartheta(1-2 \gamma)+\lambda+1}{2 \Delta(\vartheta)} \ln \{2[\lambda-1-\vartheta+\Delta(\vartheta)]\}
$$

and taking (4.14) into account, we get

$$
\mathrm{I}_{1}\left[(1-\gamma)^{-}\right]=-\frac{1}{4} \ln (\lambda)+\frac{\vartheta(1-2 \gamma)+\lambda+1}{2 \Delta(\vartheta)} \ln \{2[\lambda-1-\vartheta+\Delta(\vartheta)]\} .
$$

Using (4.12) in (4.21), we have

$$
\begin{aligned}
Q_{1}[\sigma(0)]= & \frac{1}{4} \ln \left(\frac{\rho^{2}}{\lambda}\right)+\frac{\vartheta(1-2 \gamma)+\lambda+1}{2 \Delta(\vartheta)} \\
& \times \ln \left[2 \frac{(\lambda-1-\vartheta) \rho+(\lambda+1)^{2} \gamma(1-\gamma)+(1-\lambda) \gamma(1-\gamma) \vartheta+\Delta(\vartheta) \delta}{\gamma(1-\gamma) \vartheta+\rho}\right]
\end{aligned}
$$

From (4.12) and (4.20) we get

$$
\begin{aligned}
\mathrm{I}_{1}(0)= & -\frac{1}{2} \ln \left[\frac{\rho}{\gamma(1-\gamma) \vartheta+\rho}\right]+Q_{1}[\sigma(0)] \\
= & \frac{1}{2} \ln [\gamma(1-\gamma) \vartheta+\rho]-\frac{1}{4} \ln (\lambda)+\frac{\vartheta(1-2 \gamma)+\lambda+1}{2 \Delta(\vartheta)} \\
& \times \ln \left[2 \frac{(\lambda-1-\vartheta) \rho+(\lambda+1)^{2} \gamma(1-\gamma)+(1-\lambda) \gamma(1-\gamma) \vartheta+\Delta(\vartheta) \delta}{\gamma(1-\gamma) \vartheta+\rho}\right] .
\end{aligned}
$$


Combining these results in (4.11) yields

$$
\begin{aligned}
\int_{0}^{1-\gamma-(1-\alpha) \varepsilon} \ln \left[\frac{\sigma(x)}{\vartheta+\sigma(x)}\right] d x \sim \frac{\vartheta(1-2 \gamma)+\lambda+1}{2 \Delta(\vartheta)} \ln \{2[\lambda-1-\vartheta+\Delta(\vartheta)]\} \\
-\frac{1}{2} \ln [\gamma(1-\gamma) \vartheta+\rho]-\frac{\vartheta(1-2 \gamma)+\lambda+1}{2 \Delta(\vartheta)} \\
\quad \times \ln \left[2 \frac{(\lambda-1-\vartheta) \rho+(\lambda+1)^{2} \gamma(1-\gamma)+(1-\lambda) \gamma(1-\gamma) \vartheta+\Delta(\vartheta) \delta}{\gamma(1-\gamma) \vartheta+\rho}\right] \\
=-\frac{1}{2} \ln [\gamma(1-\gamma) \vartheta+\rho]+\frac{\vartheta(1-2 \gamma)+\lambda+1}{2 \Delta(\vartheta)} \\
\quad \times \ln \left[\frac{[\gamma(1-\gamma) \vartheta+\rho][\lambda-1-\vartheta+\Delta(\vartheta)]}{(\lambda-1-\vartheta) \rho+(\lambda+1)^{2} \gamma(1-\gamma)+(1-\lambda) \gamma(1-\gamma) \vartheta+\Delta(\vartheta) \delta}\right] .
\end{aligned}
$$

From (4.12) we see that as $N \rightarrow \infty$,

$$
\frac{1}{2}\left\{\ln \left[\frac{\sigma(0)}{\vartheta+\sigma(0)}\right]+\ln \left[\frac{\sigma(1-\gamma+(\alpha-1) / N)}{\vartheta+\sigma(1-\gamma+(\alpha-1) / N)}\right]\right\} \sim \frac{1}{2} \ln \left[\frac{\rho}{\gamma(1-\gamma) \vartheta+\rho}\right] .
$$

Therefore, we conclude that

$$
\begin{aligned}
\ln [P(\vartheta)]= & \frac{1}{2} \ln \left[\frac{\rho}{\gamma(1-\gamma) \vartheta+\rho}\right]-\frac{1}{2 \varepsilon} \ln [\gamma(1-\gamma) \vartheta+\rho]+\frac{1}{\varepsilon} \frac{\vartheta(1-2 \gamma)+\lambda+1}{2 \Delta(\vartheta)} \\
& \times \ln \left[\frac{[\gamma(1-\gamma) \vartheta+\rho][\lambda-1-\vartheta+\Delta(\vartheta)]}{(\lambda-1-\vartheta) \rho+(\lambda+1)^{2} \gamma(1-\gamma)+(1-\lambda) \gamma(1-\gamma) \vartheta+\Delta(\vartheta) \delta}\right]+o(1) .
\end{aligned}
$$

Exponentiating this yields the formula for $\widetilde{P}(\vartheta ; N)$.

We note from (4.3) that

$$
\mu(\vartheta) \longrightarrow \frac{\gamma}{\delta}, \quad \vartheta \downarrow \theta_{0}
$$

and that $\widetilde{P}(\vartheta ; N)$ has a branch point singularity at $\vartheta=\theta_{0}$.

We evaluate next $h_{k}(\vartheta)$ in (3.42) for $N \rightarrow \infty$ and $k=O(N)$ with $\vartheta$ fixed. We introduce the new variables

$$
y=\frac{x}{N}, \quad z=\frac{k}{N}, \quad y \geq 0,0 \leq z \leq 1
$$


and consider the asymptotic approximation to $F_{k}(x)$ inside the domain

$$
\mathfrak{D}=\{(y, z): y \geq 0,0 \leq z \leq 1\} .
$$

Writing $h_{k}(\vartheta)$ in terms of $z$, we have

$$
\begin{aligned}
h_{k}(\vartheta) & =\frac{1}{2 \pi i} \oint_{\mathscr{C}} \frac{\left[1-R_{1}(\vartheta) w\right]^{N V(9)}\left[1+R_{2}(\vartheta) w\right]^{N[1-V(\vartheta)]}}{w^{N(1-z)+1}} d w \\
& =\frac{1}{2 \pi i} \oint_{\mathscr{C}} \frac{1}{w} \exp [N \eta(w, \vartheta, z)] d w,
\end{aligned}
$$

with

$$
\eta(w, \vartheta, z)=V(\vartheta) \ln \left[1-R_{1}(\vartheta) w\right]+[1-V(\vartheta)] \ln \left[1+R_{2}(\vartheta) w\right]-(1-z) \ln (w) .
$$

We first assume that $\gamma<z<1$ and compute the integral (4.32) as $N \rightarrow \infty$ by the saddle point method [6]. Thus, we locate the saddle points $W(\vartheta, z)$ by solving the equation

$$
\left.\frac{\partial}{\partial w} \eta(w, \vartheta, z)\right|_{w=W(\vartheta, z)}=0
$$

or

$$
z W^{2}+[(\gamma-z) \vartheta+z \lambda-z-\lambda] W+(1-z) \lambda=0
$$

and find two saddle points $W_{+}(\vartheta, z)$ and $W_{-}(\vartheta, z)$ defined by

$$
W_{ \pm}(\vartheta, z)=\frac{\vartheta+1-\lambda}{2}+\frac{1}{2 z}[\lambda-\gamma \vartheta \pm \sqrt{D(\vartheta, z)}]
$$

with

$$
D(\vartheta, z)=\rho^{2}+[2(\lambda+1) \rho+2 \phi \vartheta](z-\gamma)+\left[(\lambda+1)^{2}+2(1-\lambda) \vartheta+\vartheta^{2}\right](z-\gamma)^{2} .
$$

We next study the motion of the saddle points $W_{ \pm}(\vartheta, z)$, and also the branch points $-1 / R_{2}(\vartheta)$ and $1 / R_{1}(\vartheta)$ of $\eta(w, \vartheta, z)$, as $\vartheta$ decreases from $\infty$ to $\theta_{0}$, with $z>\gamma$. First, we observe from (3.7) that for all real $\vartheta, R_{1}(\vartheta)$ and $R_{2}(\vartheta)>0$, and thus

$$
-\frac{1}{R_{2}(\vartheta)}<0<\frac{1}{R_{1}(\vartheta)}
$$

For large 9 , we have

$$
\frac{1}{R_{1}} \sim \vartheta, \quad W_{+} \sim \frac{z-\gamma}{z} \vartheta, \quad W_{-} \sim \frac{1-z}{z-\gamma} \frac{\lambda}{\vartheta}, \quad \vartheta \longrightarrow \infty,
$$


and hence for all $\vartheta>0$,

$$
-\frac{1}{R_{2}}<0<W_{-}<W_{+}<\frac{1}{R_{1}}
$$

When $\vartheta=0$, the branch point $1 / R_{1}(\vartheta)$ coalesces with the saddle point $W_{+}$:

$$
\frac{1}{R_{1}(0)}=1, \quad W_{+}(0, z)=1, \quad W_{-}(0, z)=\frac{\lambda}{z}(1-z)
$$

and we get

$$
-\frac{1}{R_{2}(0)}<0<W_{-}(0, z)<W_{+}(0, z)=\frac{1}{R_{1}(0)}
$$

For $\theta_{0}<\vartheta<0$, the saddle point $W_{+}$moves to the right of the branch point $1 / R_{1}(\vartheta)$ :

$$
-\frac{1}{R_{2}}<0<W_{-}<\frac{1}{R_{1}}<W_{+}
$$

Finally, when $\vartheta=\theta_{0}$ with $\gamma<z<\gamma^{2} / \delta$, the branch point $1 / R_{1}(\vartheta)$ coalesces with the saddle point $W_{-}$:

$$
\frac{1}{R_{1}\left(\theta_{0}\right)}=\frac{\lambda}{\gamma}(1-\gamma), \quad W_{+}\left(\theta_{0}, z\right)=\frac{(1-z) \lambda}{(1-\gamma) z}, \quad W_{-}\left(\theta_{0}, z\right)=\frac{\lambda}{\gamma}(1-\gamma),
$$

and we have

$$
-\frac{1}{R_{2}\left(\theta_{0}\right)}<0<W_{-}\left(\theta_{0}, z\right)=\frac{1}{R_{1}\left(\theta_{0}\right)}<W_{+}\left(\theta_{0}, z\right)
$$

Note that when $\vartheta=\vartheta^{*}$, where

$$
\vartheta^{*}(z)=\frac{\lambda(z-1)-z+2 \sqrt{z \lambda(1-z)}}{z-\gamma}<\theta_{0}, \quad \gamma<z<\frac{\gamma^{2}}{\delta}
$$

the discriminant $D(\vartheta, z)$ vanishes and the saddle points coalesce. Thus,

$$
-\frac{1}{R_{2}\left(\vartheta^{*}\right)}<0<\frac{1}{R_{1}\left(\vartheta^{*}\right)}<W_{-}\left(\vartheta^{*}, z\right)=W_{+}\left(\vartheta^{*}, z\right)=\sqrt{\frac{\lambda(1-z)}{z}} .
$$

Furthermore,

$$
\frac{d}{d z} D\left(\vartheta^{*}, z\right)=\frac{\partial D}{\partial \vartheta} \frac{d \vartheta^{*}}{d z}+\frac{\partial D}{\partial z}=0, \quad \text { at } \vartheta=\vartheta^{*}
$$


since

$$
\begin{gathered}
\frac{\partial D}{\partial \vartheta}\left(\vartheta^{*}, z\right)=4(z-\gamma) \sqrt{\lambda z(1-z)}, \\
\frac{d \vartheta^{*}}{d z}=\frac{\phi \sqrt{\lambda z(1-z)}+\lambda(2 z \gamma-z-\gamma)}{\sqrt{\lambda z(1-z)}(z-\gamma)^{2}}, \\
\frac{\partial D}{\partial z}\left(\vartheta^{*}, z\right)=-\frac{4}{z-\gamma}[\phi \sqrt{\lambda z(1-z)}+\lambda(2 z \gamma-z-\gamma)] .
\end{gathered}
$$

Hence, $D(\vartheta, z)$ has a double zero at $\vartheta=\vartheta^{*}$.

From (4.40), (4.42), (4.43), and (4.45), we conclude that for $\vartheta>\theta_{0}$ with $z>\gamma$, we must deform the contour $\mathscr{C}$ to the steepest descent contour through the saddle $W_{-}$and therefore,

$$
h_{k}(\vartheta) \sim \frac{1}{\sqrt{2 \pi N}} \frac{1}{W_{-}} \exp \left[N \eta\left(W_{-}, \vartheta, z\right)\right]\left[\left.\frac{\partial^{2} \eta}{\partial w^{2}}\right|_{w=W_{-}}\right]^{-1 / 2}, \quad \vartheta>\theta_{0}, \gamma<z<1 .
$$

Note that the steepest descent directions at $w=W_{-}$are $\arg \left(w-W_{-}\right)= \pm \pi / 2$ and we can shift $\mathscr{C}$ into another circle that goes through this saddle point.

Using (4.33) and (4.35), we find that

$$
\frac{\partial^{2} \eta}{\partial w^{2}}\left(W_{ \pm}, \vartheta, z\right)=\frac{A(\vartheta, z) W_{ \pm}+B(\vartheta, z)}{\left(W_{ \pm}\right)^{2}\left[(\vartheta \gamma-\lambda) W_{ \pm}+\lambda\right]^{2}}
$$

with

$$
\begin{aligned}
A(\vartheta, z)= & \lambda^{2}(z-1)(z \lambda+z-\lambda)+\left[\left(-2 \lambda^{2}+\lambda-\gamma \lambda^{2}-\gamma\right) z^{2}+\lambda(4 \gamma \lambda+2 \lambda-\gamma) z-3 \gamma \lambda^{2}\right] \vartheta \\
& +(z-\gamma)[(2 \gamma \lambda+\lambda-2 \gamma) z-3 \gamma \lambda] \vartheta^{2}-\gamma(z-\gamma)^{2} \vartheta^{3} \\
B(\vartheta, z)= & \lambda(1-z)\left\{(z \lambda+z-\lambda) \lambda+[2 \gamma \lambda+(\gamma-\lambda-\gamma \lambda) z] \vartheta+\gamma(z-\gamma) \vartheta^{2}\right\} .
\end{aligned}
$$

Using (4.2) and (4.50) in (3.42), we have

$$
\begin{aligned}
F_{k}(x) \sim & \left(\frac{\lambda}{\lambda+1}\right)^{N} \frac{1}{\sqrt{2 \pi N}} \frac{1}{2 \pi i} \int_{\mathrm{Br}^{+}} \frac{1}{\vartheta} \frac{1}{W_{-}} \sqrt{\frac{-\theta_{0}}{\vartheta-\theta_{0}}}\left[\left.\frac{\partial^{2} \eta}{\partial w^{2}}\right|_{w=W_{-}}\right]^{-1 / 2} \\
& \times \exp \left\{N\left[y \vartheta+\mu(\vartheta)+\eta\left(W_{-}, \vartheta, z\right)\right]\right\} d \vartheta, \quad z>\gamma .
\end{aligned}
$$

To compute the above integral as $N \rightarrow \infty$, we will again use the saddle point method. We obtain $F_{k}(x) \sim G_{1}(y, z)$ with

$$
\begin{aligned}
G_{1}(y, z)= & \frac{1}{2 \pi N}\left(\frac{\lambda}{\lambda+1}\right)^{N} \frac{1}{\Theta} \frac{1}{W_{-}(\Theta, z)} \sqrt{\frac{-\theta_{0}}{\Theta-\theta_{0}}} \sqrt{\frac{1}{\eta_{w w}\left(W_{-}, \Theta, z\right)}} \\
& \times \sqrt{\frac{1}{\Psi_{\vartheta \vartheta}\left(y, W_{-}, \Theta, z\right)}} \exp \left[N \Psi\left(y, W_{-}, \Theta, z\right)\right], \quad \Theta>0, z>\gamma,
\end{aligned}
$$


where

$$
\Psi(y, w, \vartheta, z)=y \vartheta+\mu(\vartheta)+\eta(w, \vartheta, z)
$$

and $\Theta(y, z)$ is defined to be the solution of the equation

$$
0=\frac{\partial \Psi}{\partial \vartheta}\left(y, W_{-}, \Theta, z\right)=y+\mu^{\prime}(\Theta)+\frac{\partial \eta}{\partial \vartheta}\left[W_{-}(\Theta, z), \Theta, z\right]
$$

where we have used

$$
\frac{\partial \eta}{\partial w}\left[W_{-}(\Theta, z), \Theta, z\right]=0
$$

For each value of $\Theta,(4.56)$ defines implicitly a curve in the $(y, z)$-plane.

Since (4.53) has a pole at $\vartheta=0,(4.54)$ is only valid if $y$ and $z$ are such that $\Theta>0$, that is, the saddle lies to the right of the pole. Then $\mathrm{Br}^{+}$can be deformed to the steepest descent contour through $\vartheta=\Theta$. We also note that when $\Theta=0$, (4.54) is singular. We will find an asymptotic approximation valid when the saddle is close to $\vartheta=0$ in Section 6 . From (4.56), we see that $\Theta=0$ corresponds to the curve

$$
y=Y_{0}(z)=\frac{z-\gamma}{\lambda+1}-\frac{\rho}{(\lambda+1)^{2}} \ln \left(\frac{z \lambda+z-\lambda}{\rho}\right), \quad \gamma \leq z \leq 1 .
$$

For $y>Y_{0}(z)$, the saddle point $\Theta$ lies on the negative real axis to the left of the pole at $\vartheta=0$. We rewrite $(3.42)$ as

$$
F_{k}(x)-\frac{1}{(1+\lambda)^{N}}\left(\begin{array}{l}
N \\
k
\end{array}\right) \lambda^{k}=\left(\frac{\lambda}{\lambda+1}\right)^{N} \frac{1}{2 \pi i} \int_{\mathrm{Br}^{-}} e^{x \theta} \frac{1}{\vartheta}\left[\prod_{j=0}^{N-\lfloor c\rfloor-1} \frac{\theta_{j}}{\theta_{j}-\vartheta}\right] h_{k}(\vartheta) d \vartheta
$$

where $\theta_{0}<\operatorname{Re}(\vartheta)<0$ on $\mathrm{Br}^{-}$. Note that the residue at $\vartheta=0$ was already calculated in (3.28) and corresponds to $F_{k}(\infty)$. Using (4.2) and (4.50) in (4.59) and approximating the integral by the saddle point method, we obtain

$$
\begin{aligned}
& F_{k}(x)-\frac{1}{(1+\lambda)^{N}}\left(\begin{array}{c}
N \\
k
\end{array}\right) \lambda^{k} \sim \frac{1}{2 \pi N}\left(\frac{\lambda}{\lambda+1}\right)^{N} \frac{1}{\Theta} \frac{1}{W_{-}(\Theta, z)} \sqrt{\frac{-\theta_{0}}{\Theta-\theta_{0}}} \sqrt{\frac{1}{\eta_{w w}\left(W_{-}, \Theta, z\right)}} \\
& \times \sqrt{\frac{1}{\Psi_{\vartheta \vartheta}\left(y, W_{-}, \Theta, z\right)}} \exp \left[N \Psi\left(y, W_{-}, \Theta, z\right)\right], \\
& \theta_{0}<\Theta<0, \gamma<z<1,
\end{aligned}
$$

with $\Psi(y, w, \vartheta, z)$ and $\Theta(y, z)$ defined by (4.55) and (4.56), respectively. 
From (4.56), we find that the value $\Theta=\theta_{0}$ corresponds to the curve

$$
y=Y_{1}(z)=\frac{[\gamma(1-\gamma)]^{2} \rho}{\delta^{2}}\left\{\frac{\gamma^{2}-\delta z}{\gamma(1-\gamma) \rho}+\ln \left[\frac{\gamma(1-\gamma) \rho}{\gamma^{2}-\delta z}\right]-1\right\}, \quad \gamma \leq z<\frac{\gamma^{2}}{\delta} .
$$

Our approximation to the integral in (3.42) assumed that $\vartheta>\theta_{0}$. However, (4.60) passes smoothly through the curve $y=Y_{1}(z)$ since from (4.51), we have

$$
\frac{\partial^{2} \eta}{\partial w^{2}}\left(W_{-}, \vartheta, z\right) \sim \frac{\gamma^{2}\left[(1-\gamma)^{2} \lambda \gamma^{2}+\delta^{2}\left(\gamma^{2} / \delta-z\right)\right]}{\lambda^{2}(1-\gamma)^{2} \delta^{2}\left(\vartheta-\theta_{0}\right)}, \quad \vartheta \longrightarrow \theta_{0} .
$$

The discriminant $D(\Theta, z)$ vanishes when

$$
y=Y^{*}(z)=-\mu^{\prime}\left(\vartheta^{*}\right)-\frac{\partial \eta}{\partial \vartheta}\left[\sqrt{\frac{\lambda(1-z)}{z}}, \vartheta^{*}, z\right], \quad \gamma<z<\frac{\gamma^{2}}{\delta},
$$

where $9^{*}(z)$ was defined in (4.46). But, as we showed in (4.48), the derivative of the discriminant also vanishes along the curve $y=Y^{*}(z)$ so that (4.60) can be smoothly continued through this curve by simply replacing $W_{-}$by $W_{+}$.

We summarize our results below.

Theorem 4.2. Defining $y=x / N$ and $z=k / N$, for $(y, z) \in \stackrel{\circ}{\mathfrak{D}}=\{(y, z): y>0,0<z<1\}$, we have

$$
\begin{aligned}
F_{k}(x) \sim G_{1}(y, z) \equiv & \frac{1}{2 \pi N}\left(\frac{\lambda}{\lambda+1}\right)^{N} \frac{1}{\Theta} \frac{1}{W_{-}(\Theta, z)} \sqrt{\frac{-\theta_{0}}{\Theta-\theta_{0}}} \sqrt{\frac{1}{\eta_{w w}\left(W_{-}, \Theta, z\right)}} \\
& \times \sqrt{\frac{1}{\Psi_{99}\left(y, W_{-}, \Theta, z\right)}} \exp \left[N \Psi\left(y, W_{-}, \Theta, z\right)\right], \quad(y, z) \in \mathfrak{R}_{1}, \\
\mathfrak{R}_{1}= & \left\{(y, z): 0<y<Y_{0}(z), \gamma<z<1\right\}, \\
Y_{0}(z)= & \frac{z-\gamma}{\lambda+1}-\frac{\rho}{(\lambda+1)^{2}} \ln \left(\frac{z \lambda+z-\lambda}{\rho}\right), \quad \gamma \leq z \leq 1,
\end{aligned}
$$

where

$$
\begin{gathered}
\eta(w, \vartheta, z)=V(\vartheta) \ln \left[1-R_{1}(\vartheta) w\right]+[1-V(\vartheta)] \ln \left[1+R_{2}(\vartheta) w\right]-(1-z) \ln (w), \\
W_{ \pm}(\vartheta, z)=\frac{\vartheta+1-\lambda}{2}+\frac{1}{2 z}[\lambda-\gamma \vartheta \pm \sqrt{D(\vartheta, z)}], \\
D(\vartheta, z)=\rho^{2}+[2(\lambda+1) \rho+2 \phi \vartheta](z-\gamma)+\left[(\lambda+1)^{2}+2(1-\lambda) \vartheta+\vartheta^{2}\right](z-\gamma)^{2}, \\
\Psi(y, w, \vartheta, z)=y \vartheta+\mu(\vartheta)+\eta(w, \vartheta, z),
\end{gathered}
$$


and $\Theta(y, z)$ is defined to be the solution of the equation

$$
\begin{gathered}
y+\mu^{\prime}(\Theta)+\frac{\partial \eta}{\partial \vartheta}\left[W_{-}(\Theta, z), \Theta, z\right]=0, \\
\mu(\vartheta)=-\frac{1}{2} \ln [\gamma(1-\gamma) \vartheta+\rho] \\
+\frac{\vartheta(1-2 \gamma)+\lambda+1}{2 \Delta(\vartheta)} \ln \left[\frac{[\gamma(1-\gamma) \vartheta+\rho][\lambda-1-\vartheta+\Delta(\vartheta)]}{(\lambda-1-\vartheta) \rho+(\lambda+1)^{2} \gamma(1-\gamma)+(1-\lambda) \gamma(1-\gamma) \vartheta+\Delta(\vartheta) \delta}\right] .
\end{gathered}
$$

(2)

$$
\begin{gathered}
F_{k}(x)-\frac{1}{(1+\lambda)^{N}}\left(\begin{array}{c}
N \\
k
\end{array}\right) \lambda^{k} \sim G_{1}(y, z), \quad(y, z) \in \mathfrak{R}_{2}, \\
\mathfrak{R}_{2}=\left\{(y, z): Y_{0}(z)<y \leq Y^{*}(z), \quad \gamma<z<1\right\},
\end{gathered}
$$

with

$$
\begin{aligned}
& Y^{*}(z)=-\mu^{\prime}\left(\vartheta^{*}\right)-\frac{\partial \eta}{\partial \vartheta}\left[\sqrt{\frac{\lambda(1-z)}{z}}, \vartheta^{*}, z\right], \quad \gamma<z<\frac{\gamma^{2}}{\delta} \\
& \vartheta^{*}(z)=\frac{\lambda(z-1)-z+2 \sqrt{z \lambda(1-z)}}{z-\gamma}, \quad \gamma<z<\frac{\gamma^{2}}{\delta} .
\end{aligned}
$$

(3)

$$
\begin{gathered}
F_{k}(x)-\frac{1}{(1+\lambda)^{N}}\left(\begin{array}{c}
N \\
k
\end{array}\right) \lambda^{k} \sim G_{2}(y, z), \quad(y, z) \in \mathfrak{R}_{3}, \\
\mathfrak{R}_{3}=\left\{(y, z): y>Y^{*}(z), y<z<\frac{\gamma^{2}}{\delta}\right\} \cup\{(y, z): 0<y, 0<z \leq \gamma\},
\end{gathered}
$$

where

$$
\begin{aligned}
G_{2}(y, z)= & \frac{1}{2 \pi N}\left(\frac{\lambda}{\lambda+1}\right)^{N} \frac{1}{\Theta^{+}} \frac{1}{W_{+}\left(\Theta^{+}, z\right)} \sqrt{\frac{-\theta_{0}}{\Theta^{+}-\theta_{0}}} \sqrt{\frac{1}{\eta_{w w}\left(W_{+}, \Theta^{+}, z\right)}} \\
& \times \sqrt{\frac{1}{\Psi_{99}\left(y, W_{+}, \Theta^{+}, z\right)}} \exp \left[N \Psi\left(y, W_{+}, \Theta^{+}, z\right)\right]
\end{aligned}
$$

and $\Theta^{+}(y, z)$ is defined to be the solution of the equation

$$
y+\mu^{\prime}\left(\Theta^{+}\right)+\frac{\partial \eta}{\partial \vartheta}\left[W_{+}\left(\Theta^{+}, z\right), \Theta^{+}, z\right]=0 .
$$




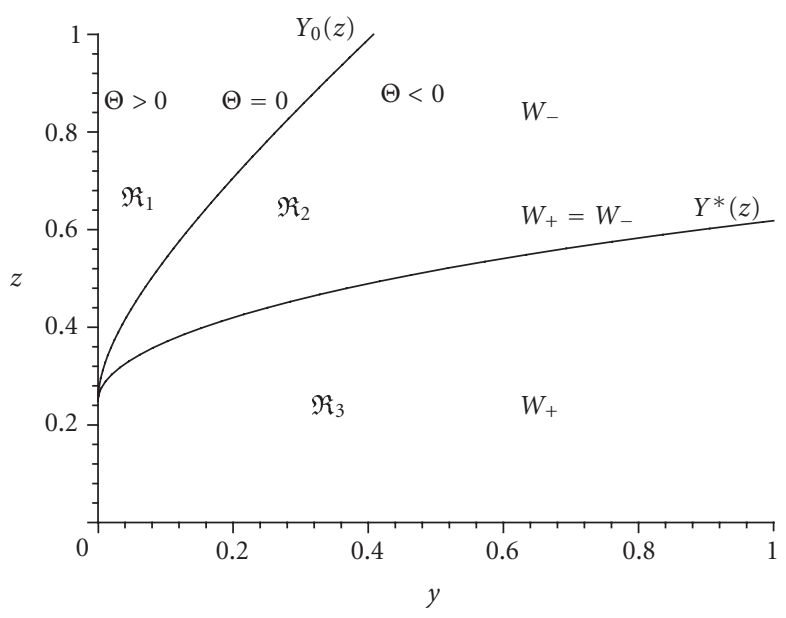

Figure 4.1. A sketch of the regions $\mathfrak{R}_{1}, \mathfrak{R}_{2}$, and $\mathfrak{R}_{3}$.

The three regions $\mathfrak{R}_{j}$ are sketched in Figure 4.1. Note that as we pass from $\mathfrak{R}_{1}$ to $\mathfrak{R}_{2}$, there is a "phase transition" in the asymptotics. However, the solution is smooth as we pass from $\mathfrak{R}_{2}$ to $\Re_{3}$.

Theorem 4.2 ceases to be valid along the curve $y=Y_{0}(z), \gamma \leq z \leq 1$, because the function $G_{1}(y, z)$ is singular there. We will find a corner layer solution near the point $(0, \gamma)$ in Section 5, a transition layer solution along $y=Y_{0}(z), \gamma<z<1$, in Section 6, and a corner layer solution near the point $\left(Y_{0}(1), 1\right)$ in Section 8.2.

From (4.36), we have

$$
\begin{aligned}
W_{-}(\vartheta, z) & \sim \frac{\lambda}{1+(1-\gamma) \vartheta}(1-z), \quad z \longrightarrow 1, \vartheta>\theta_{0}, \\
W_{+}(\vartheta, z) & \sim \frac{\lambda-\gamma \vartheta}{z}, \quad z \longrightarrow 0, \vartheta<\theta_{0},
\end{aligned}
$$

and from (4.33), we get

$$
\begin{aligned}
& \eta_{w w}\left(W_{-}, \vartheta, z\right) \sim \frac{[1+(1-\gamma) \vartheta]^{2}}{\lambda^{2}(1-z)}, \quad z \longrightarrow 1, \vartheta>\theta_{0}, \\
& \eta_{w w}\left(W_{+}, \vartheta, z\right) \sim \frac{z^{3}}{(\lambda-\gamma \vartheta)^{2}}, \quad z \longrightarrow 0, \vartheta<\theta_{0} .
\end{aligned}
$$

Hence,

$$
\begin{aligned}
& G_{1}(y, z)=O\left(\frac{1}{\sqrt{1-z}}\right), \quad z \longrightarrow 1, \\
& G_{2}(y, z)=O\left(\frac{1}{\sqrt{z}}\right), \quad z \longrightarrow 0,
\end{aligned}
$$


and our approximation develops singularities as $z \rightarrow 0$ and $z \rightarrow 1$, which corresponds to $k \approx 0$ and $k \approx N$. We will find a boundary layer solution near $z=0$ in Section 7 and a boundary layer solution near $z=1$ in Section 8 . In these cases, we will need to reexamine the expansion of (4.32).

Finally, from (4.56), we see that $\Theta \rightarrow \infty$ as $y \rightarrow 0$ if $\gamma<z<1$. We will find a boundary layer approximation near $y=0$, with $z>\gamma$ in Section 9. To analyze the case of small $y$, we will need an expansion of $P(\vartheta)$ in Lemma 4.1 valid for $\vartheta$ large. Note that Theorem 4.2 does apply for $y \rightarrow 0$ as long as $0<z<\gamma$. In this range, we can show that $\Theta^{+}$in (4.72) remains finite. In particular, the boundary masses $F_{k}(0)$ for $k=N z$ with $0<z<\gamma$ can be computed by setting $y=0$ in (4.69). We also note that in this range $F_{k}(0) \sim F_{k}(\infty)$, and the exponentially small (as $N \rightarrow \infty$ ) difference can be estimated from (4.69).

\section{The corner layer at $(0, \gamma)$ (Region I)}

We next consider the vicinity of the corner point $(y, z)=(0, \gamma)$. We first evaluate the product $P(\vartheta)$ for $N \rightarrow \infty$ and $\vartheta=O(N)$.

Lemma 5.1. Let $\vartheta=N S, S>0, S=O(1)$, and

$$
P(N S)=\mathscr{P}(S)=\prod_{j=0}^{N-\lfloor c\rfloor-1} \frac{\theta_{j}}{\theta_{j}-N S} .
$$

Then $\mathscr{P}(S) \sim \hat{P}(S ; N)$ as $N \rightarrow \infty$, where

$$
\begin{aligned}
\hat{P}(S ; N)= & \frac{1}{\sqrt{2 \pi N}} \sqrt{\frac{\rho}{\phi \gamma(1-\gamma)}}\left(\frac{\phi}{S}\right)^{\alpha} \exp \{-N(1-\gamma) \ln [(1-\gamma) S N]-N \gamma \ln (\gamma)\} \\
& \times \Gamma\left(\frac{\phi}{S}+1-\alpha\right) \exp \left\{\frac{\phi}{S} \ln \left[\frac{\gamma}{\phi(1-\gamma) N}\right]+\frac{2 \phi-\rho-1}{S}\right\} .
\end{aligned}
$$

Here, $\Gamma(\cdot)$ is the Gamma function, $\alpha=c-\lfloor c\rfloor$ is the fractional part of $c, \phi=\gamma+\lambda-\gamma \lambda$, and $\rho=\gamma \lambda+\gamma-\lambda$.

Proof. We write

$$
\begin{aligned}
\mathscr{P}(S) & =\prod_{j=0}^{(1-\gamma) N+\alpha-1} \frac{\vartheta_{j}}{\vartheta_{j}-S N} \\
& =\prod_{j=0}^{(1-\gamma) N+\alpha-1} \frac{-\sigma(j / N)}{-\sigma(j / N)-S N} \\
& =\prod_{j=0}^{(1-\gamma) N+\alpha-1} \frac{\sigma(j / N)(1-\gamma-j / N)}{[(1-\gamma) N-j] S+\sigma(j / N)(1-\gamma-j / N)} .
\end{aligned}
$$

Changing index from $j$ to $n=(1-\gamma) N+\alpha-1-j$ and defining

$$
\sigma_{n}=\sigma\left(1-\gamma+\frac{\alpha-1-n}{N}\right)
$$


we have

$$
\begin{aligned}
\mathscr{P}(S)= & \prod_{n=0}^{(1-\gamma) N+\alpha-1} \frac{((n+1-\alpha) / N) \sigma_{n}}{(n+1-\alpha) S+((n+1-\alpha) / N) \sigma_{n}} \\
= & \prod_{n=0}^{(1-\gamma) N+\alpha-1}\left[\frac{1}{\phi}\left(\frac{n+1-\alpha}{N}\right) \sigma_{n}\right] \times \prod_{n=0}^{(1-\gamma) N+\alpha-1} \frac{\phi}{\phi+(n+1-\alpha) S} \\
& \times \prod_{n=0}^{(1-\gamma) N+\alpha-1} \frac{\phi+(n+1-\alpha) S}{(n+1-\alpha) S+((n+1-\alpha) / N) \sigma_{n}} \equiv \mathscr{P}_{1}(S) \mathscr{P}_{2}(S) \mathscr{P}_{3}(S) .
\end{aligned}
$$

We evaluate individually the three products in (5.5) beginning with $\mathscr{P}_{1}(S)$.

Setting $\varepsilon=1 / \mathrm{N}$ and using the Euler-Maclaurin summation formula, we have

$$
\begin{aligned}
\ln \left[\prod_{n=0}^{(1-\gamma) N+\alpha-1} \varepsilon(n+1-\alpha) \sigma_{n}\right] \\
=\sum_{n=0}^{(1-\gamma) N+\alpha-1} \ln \left[\varepsilon(n+1-\alpha) \sigma_{n}\right] \\
=\frac{1}{2} \ln \left[(1-\gamma) \sigma_{(1-\gamma) N+\alpha-1}\right]+\frac{1}{2} \ln \left[\varepsilon(1-\alpha) \sigma_{0}\right] \\
\quad+\int_{0}^{(1-\gamma) N+\alpha-1} \ln \{\varepsilon(n+1-\alpha) \sigma[1-\gamma+(\alpha-1-n) \varepsilon]\} d n+o(1) .
\end{aligned}
$$

Changing variables to $x=1-\gamma+(\alpha-1-n) \mathcal{E}$, we get

$$
\begin{array}{r}
\int_{0}^{(1-\gamma) N+\alpha-1} \ln \{\varepsilon(n+1-\alpha) \sigma[1-\gamma+(\alpha-1-n) \varepsilon]\} d n \\
=\frac{1}{\varepsilon} \int_{0}^{1-\gamma+(\alpha-1) \varepsilon} \ln [(1-\gamma-x) \sigma(x)] d x .
\end{array}
$$

Defining

$$
\mathrm{I}_{2}(z)=\int^{z} \ln [(1-\gamma-x) \sigma(x)] d x
$$

we have

$$
\int_{0}^{1-\gamma+(\alpha-1) \mathcal{\varepsilon}} \ln [(1-\gamma-x) \sigma(x)] d x \sim \mathrm{I}_{2}(1-\gamma)-(1-\alpha) \mathrm{I}_{2}^{\prime}(1-\gamma) \mathcal{\varepsilon}-\mathrm{I}_{2}(0), \quad \varepsilon \longrightarrow 0 .
$$

From (3.14), we have

$$
(1-\gamma-x) \sigma(x)=\frac{\rho+2(\lambda-1)(1-x) x+(1-2 x) \sqrt{\rho^{2}+4 \lambda(1-x) x}}{2(\gamma-x)}
$$


and evaluating this at $x=1-\gamma$, we get

$$
\mathrm{I}_{2}^{\prime}(1-\gamma)=\ln (\phi)
$$

After some calculations, we find that

$$
\begin{aligned}
\mathrm{I}_{2}(z)= & (\gamma-z) \ln (2)-z-\ln (1-z) \\
& +\frac{\gamma}{2} \ln \left[\rho^{2}-2 \lambda(2 z \gamma-\gamma-z)+\phi K_{1}(z)\right] \\
& +\frac{1}{2}(\gamma-1) \ln \left[\rho^{2}+2 \lambda(2 z \gamma-\gamma-z+1)+\phi K_{1}(z)\right] \\
& +z \ln \left[\frac{\rho+2 z(1-z)(\lambda-1)+(1-2 z) K_{1}(z)}{\gamma-z}\right] \\
& +\frac{1}{2} \ln \left[\rho^{2}+2 \lambda(1-z)+\rho K_{1}(z)\right],
\end{aligned}
$$

where

$$
K_{1}(z)=\sqrt{\rho^{2}+4 \lambda z(1-z)} .
$$

From (5.12), we obtain

$$
\begin{aligned}
& \mathrm{I}_{2}(0)=2 \gamma \ln (2)+\frac{1}{2}(\gamma+1) \ln (\lambda+1)+\frac{\gamma}{2} \ln (\delta)+\gamma \ln (\gamma), \\
& \mathrm{I}_{2}(1-\gamma)=2 \gamma \ln (2)+\frac{1}{2}(\gamma+1) \ln (\lambda+1)+\frac{\gamma}{2} \ln (\delta)+\gamma-1 .
\end{aligned}
$$

Thus,

$$
\mathrm{I}_{2}(1-\gamma)-\mathrm{I}_{2}(0)=\gamma-1-\gamma \ln (\gamma)
$$

and we have

$$
\frac{1}{\varepsilon} \int_{0}^{1-\gamma+(\alpha-1) \varepsilon} \ln [(1-\gamma-x) \sigma(x)] d x \sim[\gamma-1-\gamma \ln (\gamma)] \frac{1}{\varepsilon}+(\alpha-1) \ln (\phi), \quad \varepsilon \longrightarrow 0 .
$$

From (3.14) and (5.4), we get

$$
\begin{aligned}
& \frac{1}{2}\left\{\ln \left[(1-\gamma) \sigma_{(1-\gamma) N+\alpha-1}\right]+\ln \left[(1-\alpha) \varepsilon \sigma_{0}\right]\right\} \\
& \quad=\frac{1}{2} \ln [(1-\gamma) \sigma(0)]+\frac{1}{2} \ln \{(1-\alpha) \varepsilon \sigma[1-\gamma+(\alpha-1) \varepsilon]\} \sim \frac{1}{2} \ln \left(\frac{\rho \phi}{\gamma}\right), \quad \varepsilon \longrightarrow 0 .
\end{aligned}
$$


Therefore, we conclude that

$$
\begin{aligned}
& \ln \left[\prod_{n=0}^{(1-\gamma) N+\alpha-1}\left(\frac{n+1-\alpha}{N}\right) \sigma_{n}\right] \\
& \quad \sim[\gamma-1-\gamma \ln (\gamma)] N+(\alpha-1) \ln (\phi)+\frac{1}{2} \ln \left(\frac{\rho \phi}{\gamma}\right), \quad N \rightarrow \infty,
\end{aligned}
$$

and then

$$
\begin{aligned}
\mathscr{P}_{1}(S) & =\prod_{n=0}^{(1-\gamma) N+\alpha-1} \frac{1}{\phi}\left(\frac{n+1-\alpha}{N}\right) \sigma_{n}=\phi^{-(1-\gamma) N-\alpha} \prod_{n=0}^{(1-\gamma) N+\alpha-1}\left(\frac{n+1-\alpha}{N}\right) \sigma_{n} \\
& \sim \sqrt{\frac{\rho}{\gamma \phi}} \exp [-(1-\gamma) \ln (\phi) N+(\gamma-1) N-\gamma \ln (\gamma) N], \quad N \longrightarrow \infty
\end{aligned}
$$

We next consider the second product in (5.5). Using properties of Gamma functions [1], we have

$$
\mathscr{P}_{2}(S)=\prod_{n=0}^{(1-\gamma) N+\alpha-1} \frac{\phi}{\phi+S(n+1-\alpha)}=\left(\frac{\phi}{S}\right)^{(1-\gamma) N+\alpha} \frac{\Gamma(\phi / S+1-\alpha)}{\Gamma[(1-\gamma) N+\phi / S+1]}
$$

and Stirling's formula [54] gives

$$
\begin{aligned}
\mathscr{P}_{2}(S) \sim & \frac{1}{\sqrt{2 \pi N}}\left(\frac{\phi}{S}\right)^{\alpha} \Gamma\left(\frac{\phi}{S}+1-\alpha\right)[(1-\gamma) N]^{-\phi / S} \frac{1}{\sqrt{1-\gamma}} \\
& \times \exp \left\{(1-\gamma) \ln \left(\frac{\phi}{S}\right) N+(1-\gamma) N-(1-\gamma)[\ln (1-\gamma) N]\right\} .
\end{aligned}
$$

Finally, we determine the asymptotic approximation to $\mathscr{P}_{3}(S)$. The Euler-Maclaurin summation formula yields

$$
\begin{aligned}
\ln \left[\mathscr{P}_{3}(S)\right]= & \sum_{n=0}^{(1-\gamma) N+\alpha-1} \ln \left[\frac{\phi+(n+1-\alpha) S}{(n+1-\alpha)\left(S+\varepsilon \sigma_{n}\right)}\right] \\
= & \frac{1}{2} \ln \left[\frac{\phi+(1-\gamma) S N}{(1-\gamma)\left(S N+\sigma_{(1-\gamma) N+\alpha-1}\right)}\right]+\frac{1}{2} \ln \left[\frac{\phi+(1-\alpha) S}{(1-\alpha)\left(S+\varepsilon \sigma_{0}\right)}\right] \\
& +\int_{0}^{(1-\gamma) N+\alpha-1} \ln \left\{\frac{\phi+(n+1-\alpha) S}{(n+1-\alpha)(S+\varepsilon \sigma[1-\gamma+(\alpha-1-n) \varepsilon])}\right\} d n+o(1) .
\end{aligned}
$$


Changing variables in the integral in (5.22) from $n$ to $x=1-\gamma+(\alpha-1-n) \mathcal{E}$, we have

$$
\begin{array}{r}
\int_{0}^{(1-\gamma) N+\alpha-1} \ln \left\{\frac{\phi+(n+1-\alpha) S}{(n+1-\alpha)(S+\varepsilon \sigma[1-\gamma+(\alpha-1-n) \varepsilon])}\right\} d n \\
\quad=\frac{1}{\varepsilon} \int_{0}^{1-\gamma+(\alpha-1) \varepsilon} \ln \left\{\frac{\phi \varepsilon+S(1-\gamma-x)}{(1-\gamma-x)[\varepsilon \sigma(x)+S]}\right\} d x .
\end{array}
$$

We write (5.23) as the difference of two integrals:

$$
\begin{aligned}
\int_{0}^{1-\gamma+(\alpha-1) \varepsilon} & \ln \left\{\frac{\phi \varepsilon+(1-\gamma-x) S}{(1-\gamma-x)[\varepsilon \sigma(x)+S]}\right\} d x \\
& =\int_{0}^{1-\gamma+(\alpha-1) \varepsilon} \ln \left[\frac{\phi \varepsilon}{1-\gamma-x}+S\right] d x-\int_{0}^{1-\gamma+(\alpha-1) \varepsilon} \ln [\varepsilon \sigma(x)+S] d x .
\end{aligned}
$$

The first integral in (5.24) is easily evaluated as $\varepsilon \rightarrow 0$ to give

$$
\begin{aligned}
\int_{0}^{1-\gamma+(\alpha-1) \varepsilon} & \ln \left[\frac{\phi \varepsilon}{1-\gamma-x}+S\right] d x \\
& =(1-\gamma) \ln (S)+\frac{\phi}{S} \ln \left\{\frac{(1-\gamma) e S}{[\phi+(1-\alpha) S] \varepsilon}\right\} \varepsilon+(1-\alpha) \ln \left[\frac{1-\alpha}{\phi+(1-\alpha) S}\right] \varepsilon+o(\varepsilon) .
\end{aligned}
$$

Introducing the function

$$
\mathrm{I}_{3}(z)=\int^{z} \ln [\varepsilon \sigma(x)+S] d x
$$

in (5.24) and using (3.6), we have

$$
\begin{aligned}
\mathrm{I}_{3}(z) & =z \ln [\varepsilon \sigma(z)+S]-\int^{z} \frac{\varepsilon x}{\varepsilon \sigma(x)+S} \sigma^{\prime}(x) d x \\
& =z \ln [\varepsilon \sigma(z)+S]-\int^{\sigma(z)} \frac{\varepsilon V(-\sigma)}{\varepsilon \sigma+S} d \sigma .
\end{aligned}
$$

After some work, we find that

$$
\begin{aligned}
\mathrm{I}_{3}(z)= & \left(z-\frac{1}{2}\right) \ln [\varepsilon \sigma(z)+S]+\frac{(2 \gamma-1) S-(\lambda+1) \varepsilon}{2 Q_{2}} \\
& \times \ln \left\{2 \frac{\Delta[-\sigma(z)] Q_{2}+[(\lambda-1) \varepsilon-S] \sigma(z)+(1-\lambda) S+(\lambda+1)^{2} \varepsilon}{\varepsilon \sigma(z)+S}\right\} \\
& +\left(\gamma-\frac{1}{2}\right) \ln \{\lambda-1+\sigma(z)+\Delta[-\sigma(z)]\}
\end{aligned}
$$


with

$$
Q_{2}(S)=\sqrt{[(\lambda+1) \varepsilon]^{2}-2(\lambda-1) \varepsilon S+S^{2}} .
$$

From (5.28), we have, as $\varepsilon \rightarrow 0$,

$$
\begin{aligned}
\mathrm{I}_{3}[1-\gamma+(\alpha-1) \varepsilon] \sim & \left(\gamma-\frac{1}{2}\right) \ln \left(\frac{8 \lambda}{S}\right)-\frac{\phi}{S} \ln \left\{\frac{4 \varepsilon \lambda[\phi+(1-\alpha) S]}{\phi S}\right\} \varepsilon \\
& +(1-\alpha) \ln \left[\frac{1-\alpha}{\phi+(1-\alpha) S}\right] \varepsilon+\frac{(2 \gamma-1)(\lambda-1)}{S} \varepsilon \\
\mathrm{I}_{3}(0) \sim & \left(\frac{1}{2}-\gamma\right) \ln \left(\frac{S}{8 \lambda}\right)-\frac{\phi}{S} \ln \left[\frac{4 \lambda(1-\gamma)}{\gamma}\right] \varepsilon+\left[\frac{1}{2}(\lambda-1)-\rho\right] \frac{\varepsilon}{S} .
\end{aligned}
$$

We conclude that

$$
\frac{1}{\varepsilon} \int_{0}^{1-\gamma+(\alpha-1) \varepsilon} \ln \left\{\frac{\phi \varepsilon+S(1-\gamma-x)}{(1-\gamma-x)[\varepsilon \sigma(x)+S]}\right\} d x \sim \frac{\phi}{S} \ln \left(\frac{\gamma}{\phi}\right)+\frac{2 \phi-\rho-1}{S} .
$$

Finally, we compute the remaining part of (5.22):

$$
\begin{aligned}
\frac{1}{2} \ln [ & \left.\frac{\phi \varepsilon+(1-\gamma) S}{(1-\gamma) S+(1-\gamma) \varepsilon \sigma_{(1-\gamma) N+\alpha-1}}\right]+\frac{1}{2} \ln \left[\frac{\phi+(1-\alpha) S}{(1-\alpha) S+(1-\alpha) \varepsilon \sigma_{0}}\right] \\
& =\frac{1}{2} \ln \left[\frac{\phi \varepsilon+(1-\gamma) S}{(1-\gamma) S+(1-\gamma) \sigma(0) \varepsilon}\right]+\frac{1}{2} \ln \left[\frac{\phi+S(1-\alpha)}{(1-\alpha) S+(1-\alpha) \varepsilon \sigma[1-\gamma+(\alpha-1) \varepsilon]}\right] \\
& \sim \frac{1}{2} \frac{\phi \gamma-\rho}{(1-\gamma) \gamma S} \varepsilon-\frac{1}{2} \frac{(1-\alpha)\left(\gamma \lambda^{2}+\gamma-4 \gamma \lambda-\lambda^{2}+2 \lambda\right)}{\phi[\phi+(1-\alpha) S]} \varepsilon=o(1) .
\end{aligned}
$$

Hence,

$$
\ln \left[\mathscr{P}_{3}(S)\right] \sim \frac{\phi}{S} \ln \left(\frac{\gamma}{\phi}\right)+\frac{2 \phi-\rho-1}{S}, \quad N \longrightarrow \infty .
$$

Combining (5.19), (5.21), and (5.33), we obtain

$$
\mathscr{P}_{1}(S) \mathscr{P}_{2}(S) \mathscr{P}_{3}(S) \sim \hat{P}(S ; N)
$$

as in (5.2). 
Next, we will analyze the function $h_{k}(\vartheta)$ for $N \rightarrow \infty$ and $(y, z)$ close to the corner $(0, \gamma)$. To do so, we introduce the new variable $l$ defined by

$$
l=k-c+\alpha, \quad-\infty<l<\infty, l=O(1),
$$

and the new function $h^{(1)}(l, S)=h_{l+c-\alpha}(S N)$. From (3.3) and (4.33), we have

$$
h^{(1)}(l, S)=\frac{1}{2 \pi i} \oint_{\mathscr{C}} \exp [\eta(w, S N, \gamma)] \frac{1}{w^{\alpha-l+1}} d w .
$$

Using (3.32), we see that, as $N \rightarrow \infty$, the branch points are located at

$$
\frac{1}{R_{1}(S N)} \sim S N, \quad-\frac{1}{R_{2}(S N)} \sim-\frac{\lambda}{S N}
$$

and that, for $N \rightarrow \infty$ with $S$ and $w$ fixed,

$$
\begin{aligned}
\exp [\eta(w, S N, \gamma)] \sim & \exp \left\{\left[(1-\gamma) N+\frac{\phi}{S}\right] \ln \left(\frac{S N}{\lambda}\right)+\frac{(1-\lambda)(1-\gamma)}{S}\right\} \\
& \times \exp \left[\frac{\lambda(1-\gamma)}{S w}-\frac{\gamma}{S} w\right] w^{\phi / S} .
\end{aligned}
$$

Therefore, we replace the contour $\mathscr{C}$ in (5.36) by a Hankel's loop and use (5.38) to obtain

$$
\begin{aligned}
h^{(1)}(l, S) \sim & \exp \left[\left(\frac{1-\gamma}{\varepsilon}+\frac{\phi}{S}\right) \ln \left(\frac{S}{\lambda \varepsilon}\right)+\frac{(1-\lambda)(1-\gamma)}{S}\right] \\
& \times \frac{1}{2 \pi i} \oint_{\mathscr{H}} \exp \left[\frac{\lambda(1-\gamma)}{S w}-\frac{\gamma}{S} w\right] w^{\phi / S+l-\alpha-1} d w,
\end{aligned}
$$

where $\mathcal{H}$ begins at $w=-\infty$, encircles $w=0$ once in a positive sense, and returns to its starting point. We assume that the branch of $w^{\phi / S+l-\alpha-1}$ takes its principal value at the point where the contour crosses the positive real axis and is continuous everywhere except for $w$ real and negative [36].

Introducing the new variable $t=(\beta / 2 \gamma)(1 / w)$, with

$$
\beta=2 \sqrt{\lambda \gamma(1-\gamma)},
$$

we get

$$
\begin{aligned}
h^{(1)}(l, S) \sim & \exp \left[\left(\frac{1-\gamma}{\varepsilon}+\frac{\phi}{S}\right) \ln \left(\frac{S}{\lambda \varepsilon}\right)+\frac{(1-\lambda)(1-\gamma)}{S}+\left(\frac{\phi}{S}+l-\alpha\right) \ln \left(\frac{\beta}{2 \gamma}\right)\right] \\
& \times \frac{1}{2 \pi i} \int_{\mathscr{H}^{\prime}} \exp \left[\frac{\beta}{2 S}\left(t-\frac{1}{t}\right)\right] t^{-\phi / S-l+\alpha-1} d t,
\end{aligned}
$$


where $\mathscr{H}^{\prime}$ is another Hankel contour of the same shape as $\mathscr{H}$. Using an integral representation of the Bessel function $J .(\cdot)$ [52], we obtain

$$
h_{k}(9)=h^{(1)}(l, S) \sim\left(\frac{S N}{\lambda}\right)^{(1-\gamma) N+\phi / S} e^{(1-\phi)(1 / S)}\left(\frac{\beta}{2 \gamma}\right)^{\phi / S+l-\alpha} J_{l-\alpha+\phi / S}\left(\frac{\beta}{S}\right) .
$$

Using (5.2) and (5.42), we obtain the following theorem.

Theorem 5.2. Let $k=c-\alpha+l=\lfloor c\rfloor+l,-\infty<l<\infty, l=O(1)$, and $x=\chi / N, \chi=O(1)$. Then $F_{k}(x) \sim F_{l}^{(1)}(\chi)$ as $N \rightarrow \infty$, where

$$
\begin{aligned}
F_{l}^{(1)}(\chi)= & \frac{1}{\sqrt{2 \pi N}} \sqrt{\frac{\rho}{\gamma \phi(1-\gamma)}}\left(\frac{\beta}{2 \gamma}\right)^{l-\alpha} \exp [N \Phi(\gamma)] \\
& \times \frac{1}{2 \pi i} \int_{\mathrm{Br}^{+}} e^{\chi S} \frac{1}{S} \Gamma\left(\frac{\phi}{S}+1-\alpha\right) J_{l-\alpha+\phi / S}\left(\frac{\beta}{S}\right) \\
& \times \exp \left\{\frac{\phi}{S} \ln \left[\frac{\beta S}{2 \lambda \phi(1-\gamma)}\right]+\frac{\phi-\rho}{S}\right\}\left(\frac{\phi}{S}\right)^{\alpha} d S,
\end{aligned}
$$

$\mathrm{Br}^{+}$is a vertical contour in the complex plane with $\operatorname{Re}(S)>0, J .(\cdot)$ is the Bessel function, $\Gamma(\cdot)$ is the Gamma function,

$$
\begin{gathered}
\beta=2 \sqrt{\lambda \gamma(1-\gamma)}, \\
\Phi(z)=-z \ln (z)-(1-z) \ln (1-z)+z \ln (\lambda)-\ln (\lambda+1) .
\end{gathered}
$$

\section{The transition layer at $y=Y_{0}(z)$ (Region II)}

As we observed previously in Section 4, the approximation (4.54) is not valid along the curve $y=Y_{0}(z)$ given by

$$
Y_{0}(z)=\frac{z-\gamma}{\lambda+1}-\frac{\rho}{(\lambda+1)^{2}} \ln \left(\frac{z \lambda+z-\lambda}{\rho}\right), \quad \gamma \leq z \leq 1 .
$$

The condition $y \approx Y_{0}(z)$ corresponds to the saddle $\Theta$ being close to the pole at $\vartheta=0$ in (3.42). To obtain the expansion of (4.54) for $y \approx Y_{0}(z)$, we first approximate the integrand in (4.53) for small $\vartheta$ using

$$
\begin{gathered}
W_{-} \sim \frac{\lambda(1-z)}{z}, \quad \eta_{w w}\left(W_{-}, \vartheta, z\right) \sim \frac{z^{3}}{\lambda^{2}(1-z)}, \\
\eta\left(W_{-}, \vartheta, z\right)+\mu(\vartheta) \sim \ln \left[(1-z)^{z-1} \lambda^{z-1} z^{-z}\right]-Y_{0}(z) \vartheta+\frac{Y_{2}(z)}{2} \vartheta^{2}, \\
Y_{2}(z)=\frac{2 \zeta}{(\lambda+1)^{4}} \ln \left(\frac{z \lambda+z-\lambda}{\rho}\right) \\
-\frac{(z-\gamma)\left[2 \rho \zeta+3(\lambda+1) \zeta(z-\gamma)+(\lambda-1)(\lambda+1)^{2}(z-\gamma)^{2}\right]}{(z \lambda+z-\lambda)^{2}(\lambda+1)^{3}}, \\
\zeta=\lambda^{2}(\gamma-1)+2 \lambda-\gamma .
\end{gathered}
$$


Using the above in (4.53) yields

$$
F_{k}(x) \sim \frac{1}{\sqrt{2 \pi N}} \sqrt{\frac{1}{z(1-z)}} \exp [\Phi(z) N] \frac{1}{2 \pi i} \int_{\mathrm{Br}^{+}} \frac{1}{\vartheta} \exp \left\{\left[y \vartheta-Y_{0}(z) \vartheta+\frac{Y_{2}(z)}{2} \vartheta^{2}\right] N\right\} d \vartheta
$$

with $\Phi(z)$ given by (5.45). Changing variables to

$$
\vartheta=\frac{v}{\sqrt{Y_{2}(z) N}}, \quad V(y, z)=\left[y-Y_{0}(z)\right] \sqrt{\frac{N}{Y_{2}(z)}},
$$

we obtain [14]

$$
\begin{aligned}
& \frac{1}{2 \pi i} \int_{\mathrm{Br}^{+}} \exp \left\{\left[y-Y_{0}(z)\right] \vartheta N+\frac{Y_{2}(z)}{2} \vartheta^{2} N\right\} \frac{1}{\vartheta} d \vartheta \\
& \quad=\frac{1}{2 \pi i} \int_{\mathrm{Br}^{\prime}} \exp \left(v V+\frac{v^{2}}{2}\right) \frac{1}{v} d v=\frac{1}{\sqrt{2 \pi}} \int_{-\infty}^{V} \exp \left(-\frac{t^{2}}{2}\right) d t
\end{aligned}
$$

where $\mathrm{Br}^{\prime}$ is another vertical contour on which $\operatorname{Re}(v)>0$. Therefore,

$$
F_{k}(x)=F^{(2)}(V, z) \sim \frac{1}{\sqrt{2 \pi N}} \sqrt{\frac{1}{z(1-z)}} \exp [\Phi(z) N] \frac{1}{\sqrt{2 \pi}} \int_{-\infty}^{V} \exp \left(-\frac{t^{2}}{2}\right) d t
$$

This is valid for $y-Y_{0}(z)=O\left(N^{-1 / 2}\right)$ with $\gamma<z<1$ and gives the transition between regions $\mathfrak{R}_{1}$ and $\mathfrak{R}_{2}$ in Theorem 4.2.

\section{The boundary $z=0$ (Region III)}

We will next analyze the part of $\partial \mathfrak{D}$ corresponding to $z=0$. We start by proving the following lemma.

Lemma 7.1. Let $k=O(1)$. Then as $N \rightarrow \infty$,

$$
h_{k}(\vartheta) \sim \frac{N^{k}}{k !}(\lambda-\gamma \vartheta)^{k}\left[-R_{1}(\vartheta)\right]^{V(\vartheta) N}\left[R_{2}(\vartheta)\right]^{[1-V(\vartheta)] N}, \quad \Theta^{+}(0,0)<\vartheta<\theta_{0},
$$

where $\Theta^{+}(y, z)$ is defined in (4.72).

Proof. From (3.5), we have for $N \rightarrow \infty$, with $w=O(N)$,

$$
H(\vartheta, w)=w^{N}\left[-R_{1}(\vartheta)\right]^{V(\vartheta) N}\left[R_{2}(\vartheta)\right]^{[1-V(\vartheta)] N} \exp \left[\frac{(\lambda-\gamma \vartheta)}{w} N+O\left(\frac{N}{w^{2}}\right)\right] .
$$

We replace $\mathscr{C}$ by $\mathscr{b}^{\prime}=\mathscr{b} / \mathrm{N}$ and change variables from $w$ to $u=w / N$ in (3.3) to get

$$
h_{k}(\vartheta)=N^{k-N} \frac{1}{2 \pi i} \oint_{\mathscr{C}} \frac{H(\vartheta, N u)}{u^{N-k+1}} d u
$$


Using (7.2), we have

$$
h_{k}(\vartheta) \sim N^{k}\left[-R_{1}(\vartheta)\right]^{V(\vartheta) N}\left[R_{2}(\vartheta)\right]^{[1-V(\vartheta)] N} \frac{1}{2 \pi i} \oint_{\mathscr{C}} \exp \left[\frac{(\lambda-\gamma \vartheta)}{u}\right] \frac{1}{u^{1-k}} d u
$$

which evaluates to give (7.1).

Writing (3.42) as

$$
F_{k}(x)-F_{k}(\infty)=\left(\frac{\lambda}{\lambda+1}\right)^{N} \frac{1}{2 \pi i} \int_{\mathrm{Br}^{-}} e^{x \vartheta} \frac{1}{\vartheta}\left[\prod_{j=0}^{N-\lfloor c \mid-1} \frac{\theta_{j}}{\theta_{j}-\vartheta}\right] h_{k}(\vartheta) d \vartheta
$$

and using (4.2) and (7.1), we have

$$
\begin{aligned}
F_{k}(x)-F_{k}(\infty) \sim & \frac{N^{k}}{k !}\left(\frac{\lambda}{\lambda+1}\right)^{N} \frac{1}{2 \pi i} \int_{\mathrm{Br}^{-}} \frac{1}{\vartheta} \sqrt{\frac{-\theta_{0}}{\vartheta-\theta_{0}}}(\lambda-\gamma \vartheta)^{k} \\
& \times \exp \left\{y \vartheta N+\mu(\vartheta) N+V(\vartheta) \ln \left[-R_{1}(\vartheta)\right] N\right. \\
& \left.+[1-V(\vartheta)] \ln \left[R_{2}(\vartheta)\right] N\right\} d \vartheta .
\end{aligned}
$$

Use of the saddle point method yields

$$
\begin{aligned}
F_{k}(x)-F_{k}(\infty) \equiv & F_{k}^{(3)}(y)-F_{k}(\infty) \sim \frac{N^{k}}{k !}\left(\frac{\lambda}{\lambda+1}\right)^{N} \frac{1}{\sqrt{2 \pi N}} \\
& \times \frac{1}{\Theta_{0}} \sqrt{\frac{-\theta_{0}}{\Theta_{0}-\theta_{0}}}\left(\lambda-\gamma \Theta_{0}\right)^{k} \exp \left[N \Psi_{0}\left(y, \Theta_{0}\right)\right]\left[\frac{\partial^{2} \Psi_{0}}{\partial \vartheta^{2}}\left(y, \Theta_{0}\right)\right]^{-1 / 2},
\end{aligned}
$$

where

$$
\Psi_{0}(y, \vartheta)=y \vartheta+\mu(\vartheta)+V(\vartheta) \ln \left[-R_{1}(\vartheta)\right]+[1-V(\vartheta)] \ln \left[R_{2}(\vartheta)\right]
$$

and $\Theta_{0}(y)$ is the solution to the equation

$$
\frac{\partial \Psi_{0}}{\partial \vartheta}\left(y, \Theta_{0}\right)=0
$$

We note from (7.9) that

$$
\begin{aligned}
& \Theta_{0}(y) \longrightarrow \Theta^{+}(0,0), \quad y \longrightarrow 0, \\
& \Theta_{0}(y) \longrightarrow \theta_{0}, \quad y \longrightarrow \infty,
\end{aligned}
$$

and thus we will limit $\Theta_{0}$ to the range $\Theta^{+}(0,0) \leq \Theta_{0}<\theta_{0}$ in (7.7). Expansion (7.7) remains valid for $y \rightarrow 0$ and can be used to evaluate $F_{k}(0)-F_{k}(\infty)$ for $k=O(1)$. 


\section{The boundary $z=1$}

We examine the scale $k=N-O(1)$ which corresponds to $1-z=O\left(N^{-1}\right)$. The analysis is different for three ranges of $y$.

\subsection{The boundary layer at $z=1,0<y<Y_{0}(1)$ (Region IV)}

Lemma 8.1. Let $k=N-j, j=O(1), \vartheta>0, \vartheta=O(1)$. Then, as $N \rightarrow \infty$,

$$
h_{k}(\vartheta) \sim \frac{N^{j}}{j !}\left[\frac{1+(1-\gamma) \vartheta}{\lambda}\right]^{j} .
$$

Proof. From (3.3), we have

$$
h_{k}(\vartheta)=\frac{1}{2 \pi i} \oint_{\mathscr{C}} \frac{H(\vartheta, w)}{w^{j+1}} d w
$$

Changing variables to $u=w N$, we get

$$
\begin{aligned}
h_{k}(\vartheta)= & N^{j} \frac{1}{2 \pi i} \oint_{\mathscr{C}^{\prime}} \exp \left\{N V(\vartheta) \ln \left[1-R_{1}(\vartheta) \frac{u}{N}\right]\right\} \\
& \times \exp \left\{N[1-V(\vartheta)] \ln \left[1+R_{2}(\vartheta) \frac{u}{N}\right]\right\} \frac{d u}{u^{j+1}} \\
\sim & N^{j} \frac{1}{2 \pi i} \oint_{\mathscr{C}^{\prime}} \exp \left[\frac{1+(1-\gamma) \vartheta}{\lambda} u\right] \frac{d u}{u^{j+1}}, \quad N \rightarrow \infty
\end{aligned}
$$

where $\mathscr{C}^{\prime}=\mathscr{C} / \mathrm{N}$ is a small loop around $u=0$. Hence, the result follows by expanding $\exp [((1+(1-\gamma) \vartheta) / \lambda) u]$ in Taylor series.

Using (4.2) and (8.1) in (3.42), we have

$$
F_{k}(x) \sim \frac{N^{j}}{j !}\left(\frac{\lambda}{\lambda+1}\right)^{N} \frac{1}{2 \pi i} \int_{\mathrm{Br}^{+}} \frac{1}{\vartheta} \sqrt{\frac{-\theta_{0}}{\vartheta-\theta_{0}}}\left[\frac{1+(1-\gamma) \vartheta}{\lambda}\right]^{j} \exp [y \vartheta N+\mu(\vartheta) N] d \vartheta .
$$

We evaluate the integral above for $N \rightarrow \infty$ by the saddle point method and obtain

$$
\begin{aligned}
F_{k}(x) \equiv & F_{j}^{(4)}(y) \sim \frac{N^{j}}{j !} \frac{1}{\sqrt{2 \pi N}}\left(\frac{\lambda}{\lambda+1}\right)^{N} \frac{1}{\Theta_{1}} \sqrt{\frac{-\theta_{0}}{\Theta_{1}-\theta_{0}}}\left[\frac{1+(1-\gamma) \Theta_{1}}{\lambda}\right]^{j} \\
& \times \exp \left[y \Theta_{1} N+\mu\left(\Theta_{1}\right) N\right] \frac{1}{\sqrt{\mu^{\prime \prime}\left(\Theta_{1}\right)}}
\end{aligned}
$$

where $\Theta_{1}(y)$ is the solution to

$$
y+\mu^{\prime}\left(\Theta_{1}\right)=0 .
$$


8.2. The corner layer at $\left(Y_{0}(1), 1\right)$ (Region $\left.\mathbf{V}\right)$. From $(8.6)$, we see that $\Theta_{1}(y) \rightarrow 0$ as $y \rightarrow$ $Y_{0}(1)$. Therefore, we will find a solution valid in the neighborhood of the point $\left(Y_{0}(1), 1\right)$. From (8.1), we have, as $N \rightarrow \infty$, with $j=O(1)$,

$$
h_{k}(\vartheta) \sim \frac{1}{j !}\left(\frac{N}{\lambda}\right)^{j}, \quad \vartheta \longrightarrow 0
$$

and from (4.3), we get

$$
\begin{aligned}
\mu(\vartheta) & \sim-\frac{\rho[\ln (\rho)-1]+1}{(\lambda+1)^{2}} \vartheta+\left[\frac{(1-\gamma)(\rho-4 \lambda+1)}{2(\lambda+1)^{3}}-\frac{\zeta \ln (\rho)}{(\lambda+1)^{4}}\right] \vartheta^{2} \\
& =-Y_{0}(1) \vartheta+\frac{1}{2} Y_{2}(1) \vartheta^{2}, \quad \vartheta \longrightarrow 0 .
\end{aligned}
$$

Using (4.2) and the above in (3.39), we obtain

$$
F_{k}(x) \sim \frac{1}{j !}\left(\frac{N}{\lambda}\right)^{j}\left(\frac{\lambda}{\lambda+1}\right)^{N} \frac{1}{2 \pi i} \int_{\mathrm{Br}^{+}} \frac{1}{\vartheta} \exp \left[y \vartheta N-Y_{0}(1) \vartheta N+\frac{1}{2} Y_{2}(1) \vartheta^{2} N\right] d \vartheta .
$$

Defining

$$
V(y, 1)=\left[y-Y_{0}(1)\right] \sqrt{\frac{N}{Y_{2}(1)}},
$$

we obtain, for $j=O(1)$ and $y-Y_{0}(1)=O\left(N^{-1 / 2}\right)$,

$$
F_{k}(x) \equiv F_{j}^{(5)}(V) \sim \frac{1}{j !}\left(\frac{N}{\lambda}\right)^{j}\left(\frac{\lambda}{\lambda+1}\right)^{N} \frac{1}{\sqrt{2 \pi}} \int_{-\infty}^{V(y, 1)} \exp \left(-\frac{t^{2}}{2}\right) d t .
$$

8.3. The boundary layer at $z=1, y>Y_{0}(1)$ (Region VI). We will examine the portion of the boundary $z=1$ for $y>Y_{0}(1)$. To do so, we write $F_{k}(x)$ as

$$
F_{k}(x)-F_{k}(\infty)=\left(\frac{\lambda}{\lambda+1}\right)^{N} \frac{1}{2 \pi i} \int_{\mathrm{Br}^{-}} e^{x \vartheta} \frac{1}{\vartheta}\left[\prod_{j=0}^{N-\lfloor c\rfloor-1} \frac{\theta_{j}}{\theta_{j}-\vartheta}\right] h_{k}(\vartheta) d \vartheta
$$

and, repeating the same calculation done in Section 8.1, we conclude that

$$
F_{k}(x)-F_{k}(\infty) \equiv F_{j}^{(6)}(y)-\left(\frac{\lambda}{1+\lambda}\right)^{N}\left(\begin{array}{c}
N \\
j
\end{array}\right) \lambda^{-j} \sim F_{j}^{(4)}(y)
$$

\section{The boundary $y=0$}

In this section, we analyze the part of the boundary where the boundary condition (2.7) applies. Theorem 4.2 is not valid for small $y$ since for $\gamma<z \leq 1$, the saddle $\Theta(y, z) \rightarrow \infty$ as $y \rightarrow 0^{+}$, but our approximation to the integrand in (3.42) was only valid for $\vartheta=O(1)$. We will scale $y=x / N$ and use Lemma 5.1 which applies for $\vartheta=S N=O(N)$. We note that $y \vartheta=x S$. We will need to examine separately the cases $\gamma<z<1$ and $1-z=O(\varepsilon)$. 


\subsection{The boundary layer at $y=0, \gamma<z<1$ (Region VII)}

LemmA 9.1. Let $k=N z, \gamma<z<1, \vartheta=S N, S>0, S=O(1)$. Then,

$$
\begin{aligned}
h_{k}(\vartheta) \sim & \frac{1}{\sqrt{2 \pi N}} \exp \left\{(1-z) \ln \left[\frac{(z-\gamma) S}{\lambda(1-z)} N\right] N+(1-\gamma) \ln \left(\frac{1-\gamma}{z-\gamma}\right) N\right\} \\
& \times \sqrt{\frac{(1-\gamma)}{(1-z)(z-\gamma)}} \exp \left[\frac{\phi}{S} \ln \left(\frac{1-\gamma}{z-\gamma}\right)+\frac{(1-z)(1-\lambda)}{S}\right] .
\end{aligned}
$$

Proof. Changing variables in (3.3) from $w$ to $u=w S N$, we have

$$
\begin{aligned}
h_{k}(\vartheta)= & \frac{1}{2 \pi i} \oint_{\mathscr{C}^{\prime}} \exp \left\{V(S N) \ln \left[1-R_{1}(S N) \frac{u}{S N}\right] N-(1-z) \ln \left(\frac{u}{S N}\right) N\right\} \\
& \times \exp \left\{[1-V(S N)] \ln \left[1+R_{2}(S N) \frac{u}{S N}\right] N\right\} \frac{d u}{u} \\
\sim & \frac{1}{2 \pi i} \oint_{\mathscr{C}^{\prime}} \exp \left[(1-z) \ln \left(\frac{S N}{u}\right) N+(1-\gamma) \ln \left(1+\frac{u}{\lambda}\right) N\right] \\
& \times \exp \left[\frac{\phi}{S} \ln \left(1+\frac{u}{\lambda}\right)+\frac{u(1-\gamma)(1-\lambda)}{(\lambda+u) S}\right] \frac{d u}{u} .
\end{aligned}
$$

Use of the saddle point method gives, for $N \rightarrow \infty$, with $S$ and $z$ fixed,

$$
\begin{aligned}
h_{k}(9) \sim & \frac{\lambda}{\sqrt{2 \pi N}} \frac{1}{u_{1}} \exp \left[(1-z) \ln \left(\frac{S N}{u_{1}}\right) N+(1-\gamma) \ln \left(1+\frac{u_{1}}{\lambda}\right) N\right] \\
& \times \exp \left[\frac{\phi}{S} \ln \left(1+\frac{u_{1}}{\lambda}\right)+\frac{u_{1}(1-\gamma)(1-\lambda)}{\left(\lambda+u_{1}\right) S}\right] \sqrt{\frac{(1-\gamma)(1-z)}{(z-\gamma)^{3}}}
\end{aligned}
$$

where the saddle point is located at

$$
u_{1}=\frac{\lambda(1-z)}{z-\gamma}
$$

Using (5.2) and (9.1) in (3.42), we get

$$
\begin{aligned}
F_{k}(x) \sim & \frac{1}{2 \pi N}\left(\frac{\lambda}{\lambda+1}\right)^{N} \sqrt{\frac{\rho}{\phi \gamma(1-z)(z-\gamma)}} \exp [-\gamma \ln (\gamma) N] \\
& \times \frac{1}{2 \pi i} \int_{\mathrm{Br}^{\prime}} \exp \left\{x S N-(1-\gamma) \ln [(z-\gamma) S N] N+(1-z) \ln \left[\frac{(z-\gamma) S}{\lambda(1-z)} N\right] N\right\} \\
& \times \exp \left\{\frac{\phi}{S} \ln \left[\frac{\gamma}{\phi(z-\gamma) N}\right]+\frac{2 \phi-\rho-1+(1-z)(1-\lambda)}{S}\right\} \\
& \times \frac{1}{S} \Gamma\left(\frac{\phi}{S}+1-\alpha\right)\left(\frac{\phi}{S}\right)^{\alpha} d S
\end{aligned}
$$


where $\mathrm{Br}^{\prime}$ is a vertical contour on which $\operatorname{Re}(S)>0$. Computing the integral in (9.5) as $N \rightarrow \infty$ by the saddle point method, we find that the integrand has a saddle at

$$
S^{*}(x, z)=\frac{z-\gamma}{x}
$$

and hence the leading term for (9.5) is

$$
\begin{aligned}
F_{k}(x) \sim & \left(\frac{1}{2 \pi N}\right)^{3 / 2}\left(\frac{\lambda}{\lambda+1}\right)^{N} \sqrt{\frac{\rho}{\phi \gamma(1-z)}} \frac{1}{z-\gamma} \exp [-\gamma \ln (\gamma) N] \\
& \times \exp \left\{(z-\gamma) N-(1-\gamma) \ln \left[\frac{(z-\gamma)^{2}}{x} N\right] N+(1-z) \ln \left[\frac{(z-\gamma)^{2}}{\lambda(1-z) x} N\right] N\right\} \\
& \times \exp \left\{\frac{x \phi}{z-\gamma} \ln \left[\frac{\gamma}{\phi(z-\gamma) N}\right]+\frac{2 \phi-\rho-1+(1-z)(1-\lambda)}{z-\gamma} x\right\} \\
& \times \Gamma\left(\frac{x \phi}{z-\gamma}+1-\alpha\right)\left(\frac{x \phi}{z-\gamma}\right)^{\alpha} .
\end{aligned}
$$

After some simplification, we get

$$
\begin{aligned}
F_{k}(x) \equiv & F^{(7)}(x, z) \sim\left(\frac{1}{2 \pi N}\right)^{3 / 2} \sqrt{\frac{\rho}{\phi \gamma(1-z)}} \frac{1}{z-\gamma} \Gamma\left(\frac{x \phi}{z-\gamma}+1-\alpha\right)\left(\frac{x \phi}{z-\gamma}\right)^{\alpha} \\
& \times \exp \left\{(z-\gamma) \ln \left[\frac{x e}{(z-\gamma)^{2} N}\right] N+z \ln (\lambda) N\right\} \\
& \times \exp [-(1-z) \ln (1-z) N-\ln (\lambda+1) N-\gamma \ln (\gamma) N] \\
& \times \exp \left\{\frac{x \phi}{z-\gamma} \ln \left[\frac{\gamma}{\phi(z-\gamma) N}\right]+\left[\frac{2 \lambda(1-\gamma)}{z-\gamma}+(\lambda-1)\right] x\right\} .
\end{aligned}
$$

Note that (9.8) is singular as $z \rightarrow \gamma$. We can show that if we expand $(9.8)$ as $(x, z) \rightarrow(0, \gamma)$, with $x /(z-\gamma)$ fixed, the result asymptotically matches the corner layer approximation in Theorem 5.2. The latter must be expanded for $\chi, l \rightarrow \infty$ with $l / \chi$ fixed.

9.2. The corner layer at $(0,1)$ (Region VIII). When $z \rightarrow 1,(9.8)$ becomes singular. Therefore, we will find an approximation to $F_{k}(x)$ close to the point $(0,1)$. We first observe that when

$$
k=N-j, \quad \vartheta=S N, \quad S>0, \quad j, S=O(1)
$$

we obtain from (3.3), by a calculation similar to that in the proof of Lemma 8.1,

$$
h_{k}(\vartheta) \sim N^{2 j} \frac{1}{j !}\left[\frac{(1-\gamma) S}{\lambda}\right]^{j} .
$$


Using (5.2) and (9.10) in (3.39), we have

$$
\begin{aligned}
F_{k}(x) \sim & N^{2 j} \frac{1}{j !}\left(\frac{\lambda}{\lambda+1}\right)^{N} \frac{1}{\sqrt{2 \pi N}} \sqrt{\frac{\rho}{\phi \gamma(1-\gamma)}} \\
& \times \frac{1}{2 \pi i} \int_{\mathrm{Br}^{+}} \exp \{x S N-(1-\gamma) \ln [(1-\gamma) S N] N-\gamma \ln (\gamma) N\} \\
& \times \frac{1}{S}\left(\frac{\phi}{S}\right)^{\alpha} \Gamma\left(\frac{\phi}{S}+1-\alpha\right)\left[\frac{(1-\gamma) S}{\lambda}\right]^{j} \\
& \times \exp \left\{\frac{\phi}{S} \ln \left[\frac{\gamma}{\phi(1-\gamma) N}\right]+\frac{2 \phi-\rho-1}{S}\right\} d S .
\end{aligned}
$$

Using the saddle point method, we find that the integrand of (9.11) has a saddle at

$$
S^{*}(x, 1)=\frac{1-\gamma}{x}
$$

where $S^{*}(x, z)$ was defined in (9.6). Hence, to leading order, we obtain

$$
\begin{aligned}
F_{k}(x) \equiv & F_{j}^{(8)}(x) \sim N^{2 j} \frac{1}{j !}\left(\frac{\lambda}{\lambda+1}\right)^{N} \frac{1}{2 \pi N} \sqrt{\frac{\rho}{\phi \gamma}} \frac{1}{1-\gamma} \\
& \times \exp \left\{(1-\gamma) \ln \left[\frac{e x}{(1-\gamma)^{2} N}\right] N-\gamma \ln (\gamma) N\right\} \\
& \times\left(\frac{x \phi}{1-\gamma}\right)^{\alpha} \Gamma\left(\frac{x \phi}{1-\gamma}+1-\alpha\right)\left[\frac{(1-\gamma)^{2}}{\lambda x}\right]^{j} \\
& \times \exp \left\{\frac{x \phi}{1-\gamma} \ln \left[\frac{\gamma}{\phi(1-\gamma) N}\right]+(3 \lambda-1) x\right\} .
\end{aligned}
$$

Note that from (9.8) and (9.13), we have

$$
F_{k}(x)=O\left(x^{\alpha+(z-\gamma) N}\right)=O\left(x^{k-\lfloor c\rfloor}\right), \quad x \longrightarrow 0,\lfloor c\rfloor+1 \leq k \leq N
$$

and thus we recover the result found in [3, equation (38)] that

$$
\frac{d^{n} F_{k}}{d x^{n}}(0)=0, \quad\lfloor c\rfloor+1+n \leq k \leq N
$$

\section{Summary and numerical studies}

In most of the strip $\mathfrak{D}=\{(y, z): y \geq 0,0 \leq z \leq 1\}$, the asymptotic expansion of $F_{k}(x)$ is given by Theorem 4.2. Below we summarize our results for the various boundary, corner, 


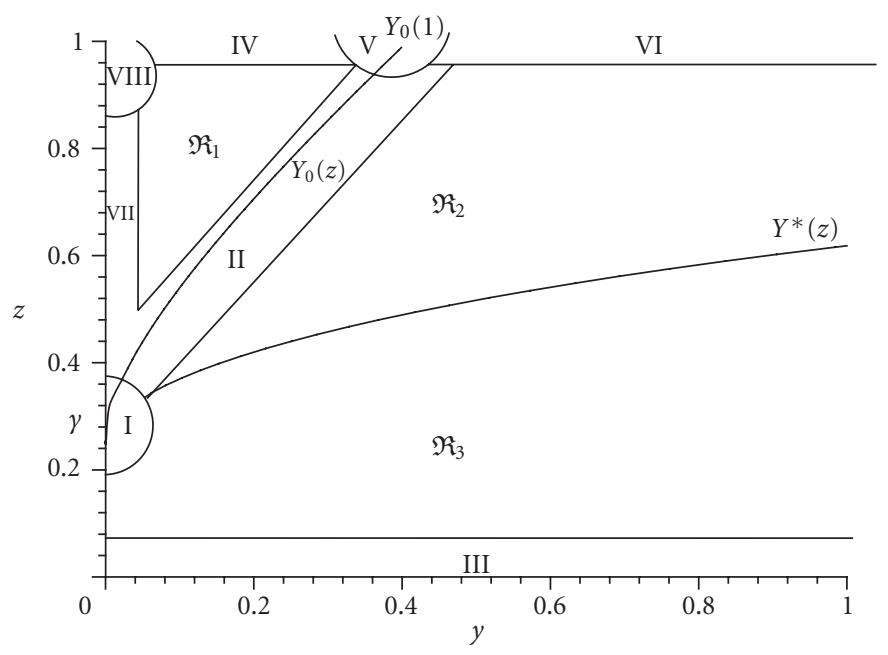

Figure 10.1. A sketch of the different asymptotic regions.

and transition layer regions where Theorem 4.2 does not apply. The paragraph number refers to the corresponding region (see Figure 10.1).

Theorem 10.1. As $N \rightarrow \infty$, asymptotic approximations of the joint distribution $F_{k}(x)$ are as follows:

(I) $k=c-\alpha+l=\lfloor c\rfloor+l,-\infty<l<\infty, l=O(1)$, and $x=\chi / N, \chi=O(1), F_{k}(x) \sim$ $F_{l}^{(1)}(\chi)$ as $N \rightarrow \infty$, where

$$
\begin{aligned}
F_{l}^{(1)}(\chi)= & \frac{1}{\sqrt{2 \pi N}} \sqrt{\frac{\rho}{\gamma \phi(1-\gamma)}}\left(\frac{\beta}{2 \gamma}\right)^{l-\alpha} \exp [N \Phi(\gamma)] \\
& \times \frac{1}{2 \pi i} \int_{\mathrm{Br}^{+}} e^{\chi S} \frac{1}{S} \Gamma\left(\frac{\phi}{S}+1-\alpha\right) J_{l-\alpha+\phi / S}\left(\frac{\beta}{S}\right) \\
& \times \exp \left\{\frac{\phi}{S} \ln \left[\frac{\beta S}{2 \lambda \phi(1-\gamma)}\right]+\frac{\phi-\rho}{S}\right\}\left(\frac{\phi}{S}\right)^{\alpha} d S,
\end{aligned}
$$

$\mathrm{Br}^{+}$is a vertical contour in the complex plane with $\operatorname{Re}(S)>0, J .(\cdot)$ is the Bessel function, $\Gamma(\cdot)$ is the Gamma function,

$$
\begin{gathered}
\beta=2 \sqrt{\lambda \gamma(1-\gamma)} \\
\Phi(z)=-z \ln (z)-(1-z) \ln (1-z)+z \ln (\lambda)-\ln (\lambda+1) ;
\end{gathered}
$$

(II) $y-Y_{0}(z)=O\left(N^{-1 / 2}\right), \gamma<z<1$ :

$$
F_{k}(x)=F^{(2)}(V, z) \sim \frac{1}{\sqrt{2 \pi N}} \sqrt{\frac{1}{z(1-z)}} \exp [\Phi(z) N] \frac{1}{\sqrt{2 \pi}} \int_{-\infty}^{V} \exp \left(-\frac{t^{2}}{2}\right) d t
$$


with

$$
\begin{aligned}
V(y, z)= & \frac{y-Y_{0}(z)}{\sqrt{Y_{2}(z)}} \sqrt{N} \\
Y_{0}(z)= & \frac{z-\gamma}{\lambda+1}-\frac{\rho}{(\lambda+1)^{2}} \ln \left(\frac{z \lambda+z-\lambda}{\rho}\right), \quad \gamma<z<1, \\
Y_{2}(z)= & \frac{2 \zeta}{(\lambda+1)^{4}} \ln \left(\frac{z+z \lambda-\lambda}{\rho}\right) \\
& -\frac{z-\gamma}{(\lambda+1)(\lambda z+z-\lambda)^{2}}\left[\frac{2 \zeta \rho}{(\lambda+1)^{2}}+\frac{3 \zeta}{(\lambda+1)}(z-\gamma)+(\lambda-1)(z-\gamma)^{2}\right], \\
\zeta= & 2 \lambda-\gamma+(\gamma-1) \lambda^{2} ;
\end{aligned}
$$

(III) $k=O(1)$ :

$$
\begin{aligned}
F_{k}(x)-F_{k}(\infty)= & F_{k}^{(3)}(y)-F_{k}(\infty) \sim \frac{N^{k}}{k !}\left(\frac{\lambda}{\lambda+1}\right)^{N} \frac{1}{\sqrt{2 \pi N}} \\
& \times \frac{1}{\Theta_{0}} \sqrt{\frac{-\theta_{0}}{\Theta_{0}-\theta_{0}}}\left(\lambda-\gamma \Theta_{0}\right)^{k} \exp \left[N \Psi_{0}\left(y, \Theta_{0}\right)\right]\left[\frac{\partial^{2} \Psi_{0}}{\partial \vartheta^{2}}\left(y, \Theta_{0}\right)\right]^{-1 / 2},
\end{aligned}
$$

where

$$
\Psi_{0}(y, \vartheta)=y \vartheta+\mu(\vartheta)+V(\vartheta) \ln \left[-R_{1}(\vartheta)\right]+[1-V(\vartheta)] \ln \left[R_{2}(\vartheta)\right]
$$

and $\Theta_{0}(y)$ is the solution to the equation

$$
\frac{\partial \Psi_{0}}{\partial \vartheta}\left(y, \Theta_{0}\right)=0
$$

(IV) $k=N-j, j=O(1), 0<y<Y_{0}(1)$ :

$$
\begin{aligned}
F_{k}(x)= & F_{j}^{(4)}(y) \sim \frac{N^{j}}{j !} \frac{1}{\sqrt{2 \pi N}}\left(\frac{\lambda}{\lambda+1}\right)^{N} \frac{1}{\Theta_{1}} \sqrt{\frac{-\theta_{0}}{\Theta_{1}-\theta_{0}}}\left[\frac{1+(1-\gamma) \Theta_{1}}{\lambda}\right]^{j} \\
& \times \exp \left[y \Theta_{1} N+\mu\left(\Theta_{1}\right) N\right] \frac{1}{\sqrt{\mu^{\prime \prime}\left(\Theta_{1}\right)}}
\end{aligned}
$$

where $\Theta_{1}(y)$ is the solution to

$$
y+\mu^{\prime}\left(\Theta_{1}\right)=0
$$

(V) $k=N-j, j=O(1), y-Y_{0}(1)=O\left(N^{-1 / 2}\right)$ :

$$
F_{k}(x)=F_{j}^{(5)}(V) \sim \frac{1}{j !}\left(\frac{N}{\lambda}\right)^{j}\left(\frac{\lambda}{\lambda+1}\right)^{N} \frac{1}{\sqrt{2 \pi}} \int_{-\infty}^{V(y, 1)} \exp \left(-\frac{t^{2}}{2}\right) d t,
$$


with

$$
V(y, 1)=\left[y-Y_{0}(1)\right] \sqrt{\frac{N}{Y_{2}(1)}}
$$

(VI) $k=N-j, j=O(1), y>Y_{0}(1)$ :

$$
F_{k}(x)-F_{k}(\infty)=F_{j}^{(6)}(y)-\left(\frac{\lambda}{1+\lambda}\right)^{N}\left(\begin{array}{c}
N \\
j
\end{array}\right) \lambda^{-j} \sim F_{j}^{(4)}(y),
$$

where $F_{j}^{(4)}(y)$ is as in item $(I V)$;

(VII) $x=O(1), y<z<1$ :

$$
\begin{aligned}
F_{k}(x)= & F^{(7)}(x, z) \sim\left(\frac{1}{2 \pi N}\right)^{3 / 2} \sqrt{\frac{\rho}{\phi \gamma(1-z)}} \frac{1}{z-\gamma} \Gamma\left(\frac{x \phi}{z-\gamma}+1-\alpha\right) \\
& \times\left(\frac{x \phi}{z-\gamma}\right)^{\alpha} \exp \left\{(z-\gamma) \ln \left[\frac{x e}{(z-\gamma)^{2} N}\right] N+z \ln (\lambda) N\right\} \\
& \times \exp [-(1-z) \ln (1-z) N-\ln (\lambda+1) N-\gamma \ln (\gamma) N] \\
& \times \exp \left\{\frac{x \phi}{z-\gamma} \ln \left[\frac{\gamma}{\phi(z-\gamma) N}\right]+\left[\frac{2 \lambda(1-\gamma)}{z-\gamma}+(\lambda-1)\right] x\right\} ;
\end{aligned}
$$

(VIII) $k=N-j, j=O(1), x=O(1)$ :

$$
\begin{aligned}
F_{k}(x)= & F_{j}^{(8)}(x) \sim N^{2 j} \frac{1}{j !}\left(\frac{\lambda}{\lambda+1}\right)^{N} \frac{1}{2 \pi N} \sqrt{\frac{\rho}{\phi \gamma}} \frac{1}{1-\gamma} \\
& \times \exp \left\{(1-\gamma) \ln \left[\frac{e x}{(1-\gamma)^{2} N}\right] N-\gamma \ln (\gamma) N\right\} \\
& \times\left(\frac{x \phi}{1-\gamma}\right)^{\alpha} \Gamma\left(\frac{x \phi}{1-\gamma}+1-\alpha\right)\left[\frac{(1-\gamma)^{2}}{\lambda x}\right]^{j} \\
& \times \exp \left\{\frac{x \phi}{1-\gamma} \ln \left[\frac{\gamma}{\phi(1-\gamma) N}\right]+(3 \lambda-1) x\right\} .
\end{aligned}
$$

From (3.42), we see that the continuous part of the density (for $x>0$ ) is given by

$$
f_{k}(x) \equiv F_{k}^{\prime}(x)=\left(\frac{\lambda}{\lambda+1}\right)^{N} \frac{1}{2 \pi i} \int_{\mathrm{Br}^{+}} e^{x \vartheta}\left[\prod_{j=0}^{N-\lfloor c \mid-1} \frac{\theta_{j}}{\theta_{j}-\vartheta}\right] h_{k}(\vartheta) d \vartheta .
$$




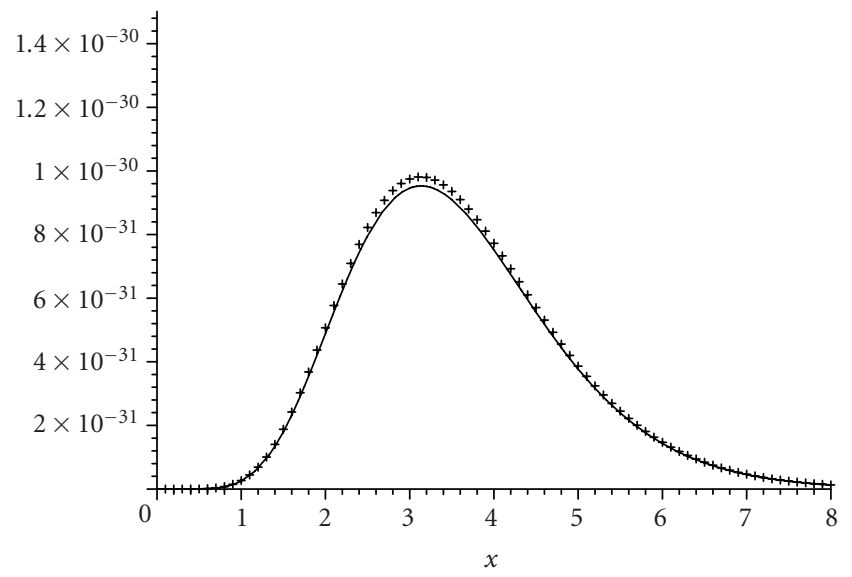

Figure 10.2. A comparison of the exact (solid curve) and asymptotic (+++) values of the density $f_{k}(x)$ for $k=17$ with $N=20$.

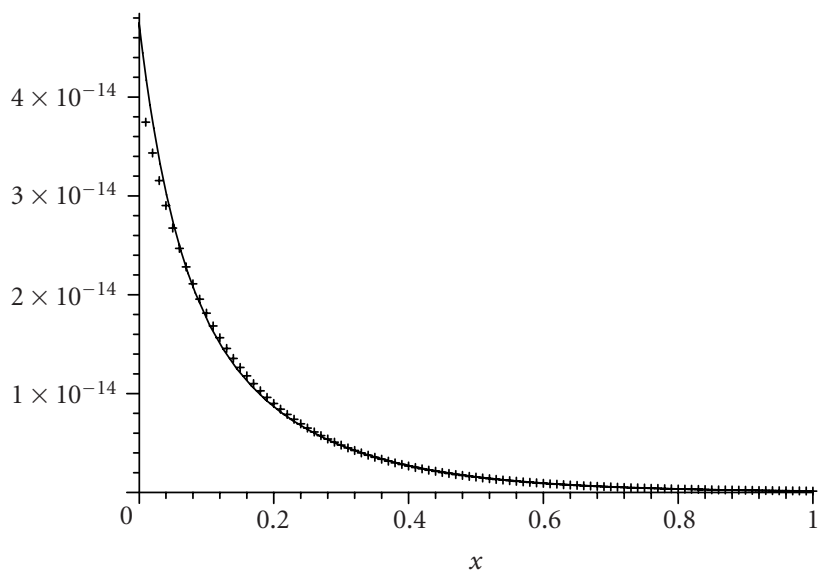

Figure 10.3. A comparison of the exact (solid curve) and asymptotic $(+++)$ values of the density $f_{k}(x)$ for $k=3$ with $N=20$.

Therefore, we can approximate the density by multiplying the asymptotic approximations by the corresponding saddle point:

$$
\begin{gathered}
f_{k}(x) \sim \Theta G_{1}(y, z), \quad f_{k}(x) \sim \Theta^{+} G_{2}(y, z), \quad f_{k}(x) \sim \Theta_{0}\left[F_{k}^{(3)}(y)-F_{k}(\infty)\right], \\
f_{k}(x) \sim \Theta_{1} F_{j}^{(4)}(y), \quad f_{k}(x) \sim S^{*}(x, z) N F^{(7)}(x, z) .
\end{gathered}
$$

Here, the right-hand sides are the expansions valid in the various regions.

We compare our asymptotic approximations to the density with the exact results obtained by evaluating (10.15). We use $N=20, \gamma=0.37987897$, and $\lambda=0.0122448$. In Figure 10.2, we graph $f_{k}(x)$ and the asymptotic approximation $\Theta G_{1}(y, z)$ for $k=17$. 


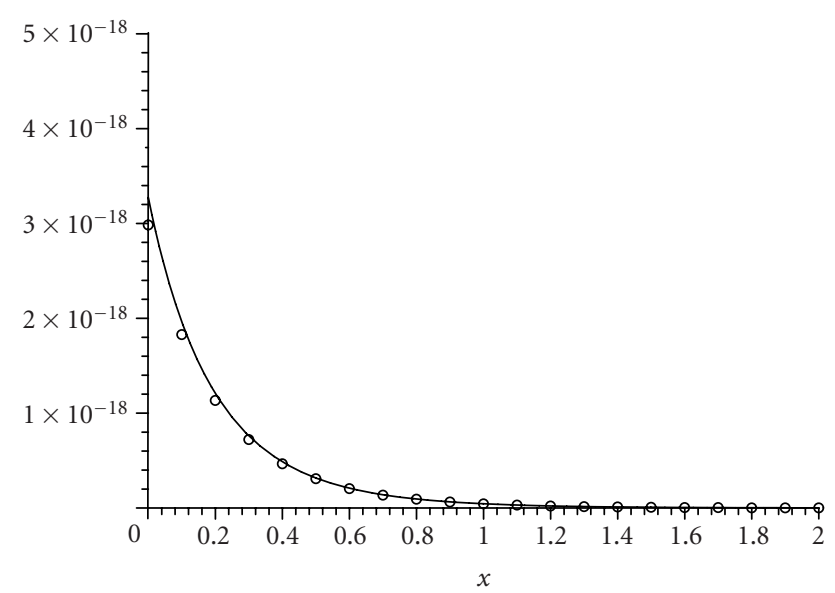

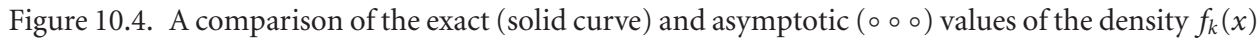
for $k=0$ with $N=20$.

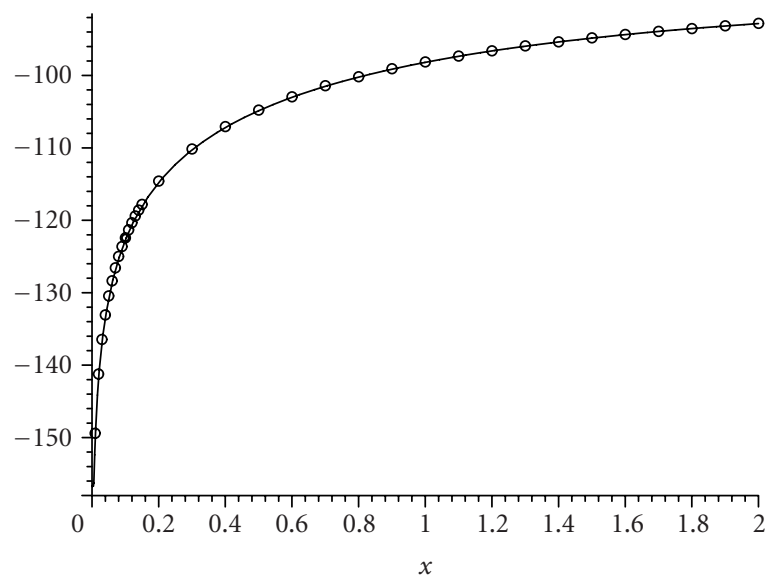

Figure 10.5. A comparison of the exact (solid curve) and asymptotic (०००) values of $\ln \left[f_{k}(x)\right]$ for $z=1$ with $N=20$.

We note that the asymptotics predict that the maximum value will be achieved at $x=$ $N Y_{0}(k / N) \simeq 3.052$.

In Figure 10.3, we graph $f_{3}(x)$ and the asymptotic approximation $\Theta^{+} G_{2}(y, z)$. We note that the maximum is now achieved at $x=0$, which is consistent with the asymptotic analysis.

In Figure 10.4, we graph $f_{0}(x)$ and $\Theta_{0}\left[F_{0}^{(3)}(y)-F_{0}(\infty)\right]$. This shows the importance of treating Region III.

In Figure 10.5, we graph $\ln \left[f_{20}(x)\right]$ and $\ln \left[\Theta_{1} F_{0}^{(4)}(y)\right]$, the latter being the boundary layer approximation to the density at $z=1$. 


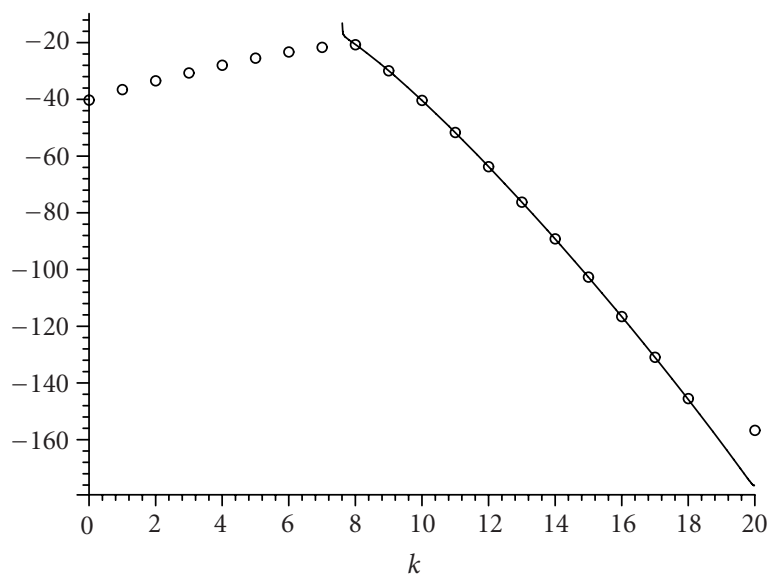

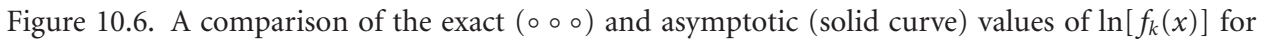
$x=0.001$ with $N=20$.

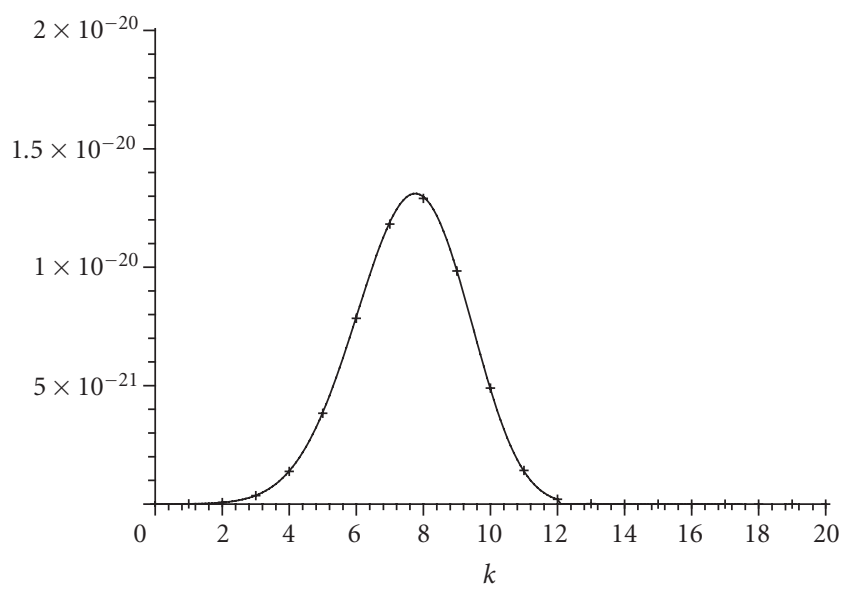

Figure 10.7. A comparison of the exact $(+++)$ and asymptotic (solid curve) values of the density $f_{k}(x)$ for $x=5$ with $N=20$.

In Figure 10.6, we graph $\ln \left[f_{k}(x)\right]$ and $\ln \left[S^{*}(x, z) N F^{(7)}(x, z)\right]$, with $x=0.001$. We see how the asymptotic approximation breaks down for $z \approx \gamma$ and for $z \approx 1$ (it does not apply for $z<\gamma)$.

In Figure 10.7, we graph $f_{k}(x)$ and the asymptotic approximations $\Theta G_{1}(y, z)$ and $\Theta^{+} G_{2}(y, z)$, with $x=5$. To show in more detail the different ranges, in Table 10.1 we compare the exact value of the density with the approximations given by $\Theta_{0} F_{k}^{(3)}(y)$ and $\Theta^{+} G_{2}(y, z)$ for $x=1(y=0.05)$, with $0 \leq k \leq 9$. In Table 10.2, we compare $f_{k}(x)$ with the approximations given by $\Theta_{1} F_{j}^{(4)}(y)$ and $\Theta G_{1}(y, z)$ for $x=1(y=0.05)$, with $10 \leq k \leq N$. We note that for our choice of parameters, $c \simeq 7.598$ and $Y_{0}(1) \simeq .4998$. Also, when $y=1 / N=0.05$, the equation $Y^{*}(z)=y$ has the solution $z \simeq 0.4811$ so that the transition between $\mathfrak{R}_{2}$ and $\mathfrak{R}_{3}$ occurs at $N z \simeq 9.622$. 
Table 10.1

\begin{tabular}{ccccc}
\hline$k$ & $\Theta$ & Exact & $\Theta_{0} F_{k}^{(3)}(y)$ & $\Theta^{+} G_{2}(y, z)$ \\
\hline 0 & -3.418 & $0.4454 \times 10^{-19}$ & $0.4324 \times 10^{-19}$ & - \\
1 & -3.593 & $0.1255 \times 10^{-17}$ & $0.1133 \times 10^{-17}$ & $0.1334 \times 10^{-17}$ \\
2 & -3.785 & $0.1686 \times 10^{-16}$ & $0.1486 \times 10^{-16}$ & $0.1727 \times 10^{-16}$ \\
3 & -3.994 & $0.1425 \times 10^{-15}$ & $0.1298 \times 10^{-15}$ & $0.1415 \times 10^{-15}$ \\
4 & -4.216 & $0.8355 \times 10^{-15}$ & $0.8503 \times 10^{-15}$ & $0.8187 \times 10^{-15}$ \\
5 & -4.444 & $0.3522 \times 10^{-14}$ & $0.4459 \times 10^{-14}$ & $0.3501 \times 10^{-14}$ \\
6 & -4.660 & $0.1062 \times 10^{-13}$ & $0.1947 \times 10^{-13}$ & $0.1010 \times 10^{-13}$ \\
7 & -4.825 & $0.2196 \times 10^{-13}$ & - & $0.2073 \times 10^{-13}$ \\
8 & -4.838 & $0.2833 \times 10^{-13}$ & - & $0.2707 \times 10^{-13}$ \\
9 & -4.411 & $0.1826 \times 10^{-13}$ & - & $0.1648 \times 10^{-13}$ \\
\hline
\end{tabular}

Table 10.2

\begin{tabular}{ccccc}
\hline$k$ & $\Theta$ & Exact & $\Theta G_{1}(y, z)$ & $\Theta_{1} F_{j}^{(4)}(y)$ \\
\hline 10 & -3.177 & $0.2389 \times 10^{-14}$ & $0.2297 \times 10^{-14}$ & - \\
11 & -1.650 & $0.7266 \times 10^{-16}$ & $0.7400 \times 10^{-16}$ & - \\
12 & -0.2175 & $0.8578 \times 10^{-18}$ & $0.8635 \times 10^{-18}$ & - \\
13 & 1.115 & $0.4972 \times 10^{-20}$ & $0.5074 \times 10^{-20}$ & - \\
14 & 2.378 & $0.1599 \times 10^{-22}$ & $0.1646 \times 10^{-22}$ & - \\
15 & 3.591 & $0.3044 \times 10^{-25}$ & $0.3145 \times 10^{-25}$ & $0.1467 \times 10^{-27}$ \\
16 & 4.769 & $0.3540 \times 10^{-28}$ & $0.3740 \times 10^{-28}$ & $0.5326 \times 10^{-31}$ \\
17 & 5.921 & $0.2519 \times 10^{-31}$ & $0.2650 \times 10^{-31}$ & $0.1449 \times 10^{-34}$ \\
18 & 7.052 & $0.1063 \times 10^{-34}$ & $0.1143 \times 10^{-34}$ & $0.2630 \times 10^{-38}$ \\
19 & 8.167 & $0.2422 \times 10^{-38}$ & $0.2422 \times 10^{-38}$ & $0.2386 \times 10^{-42}$ \\
20 & 9.269 & $0.2284 \times 10^{-42}$ & - & \\
\hline
\end{tabular}

\section{Conditional limit laws}

In this section, we will study the probability of $k$ sources being on, given that the buffer content is equal to $x>0$, and the probability of the buffer having some nonzero content $x$, given that $k$ sources are on, for various ranges of $x$ and $k$.

11.1. The distribution of active sources conditioned on $x$. We define

$$
\begin{aligned}
& M_{1}(x) \equiv \sum_{k=0}^{N} f_{k}(x)=\lim _{t \rightarrow \infty} \frac{d}{d x} \operatorname{Pr}[X(t) \leq x], \quad x>0, \\
& M_{2}(k) \equiv \int_{0}^{\infty} f_{k}(x) d x=\lim _{t \rightarrow \infty} \operatorname{Pr}[Z(t)=k, X(t)>0], \quad 0 \leq k \leq N,
\end{aligned}
$$


and the conditional probabilities

$$
\begin{aligned}
& f(k \mid x)=\frac{f_{k}(x)}{M_{1}(x)}=\lim _{t \rightarrow \infty} \operatorname{Pr}[Z(t)=k \mid X(t)=x>0], \quad x>0,0 \leq k \leq N, \\
& f(x \mid k)=\frac{f_{k}(x)}{M_{2}(k)}=\lim _{t \rightarrow \infty} \frac{d}{d x} \operatorname{Pr}[X(t) \leq x \mid Z(t)=k, X(t)>0], \quad x>0,0 \leq k \leq N .
\end{aligned}
$$

Note that

$$
M_{2}(k)=F_{k}(\infty)-F_{k}(0), \quad 0 \leq k \leq N
$$

We first consider $x=O(N)$. In this range, we compute $M_{1}(x)$ using the approximation in $\mathfrak{R}_{3}$ :

$$
\begin{aligned}
\sum_{k=0}^{N} f_{k}(x) \sim & \frac{1}{2 \pi}\left(\frac{\lambda}{\lambda+1}\right)^{N} \int_{0}^{1} \frac{1}{W_{+}\left(\Theta^{+}, z\right)} \sqrt{\frac{-\theta_{0}}{\Theta^{+}-\theta_{0}}} \sqrt{\frac{1}{\eta_{w w}\left(W_{+}, \Theta^{+}, z\right)}} \\
& \times \sqrt{\frac{1}{\Psi_{\vartheta \vartheta}\left(y, W_{+}, \Theta^{+}, z\right)}} \exp \left[N \Psi\left(y, W_{+}, \Theta^{+}, z\right)\right] d z .
\end{aligned}
$$

The main contribution to the integral comes from near $z=\gamma$, and by Laplace's method we have

$$
\begin{aligned}
\sum_{k=0}^{N} f_{k}(x) \sim & \frac{1}{\sqrt{2 \pi N}\left(\frac{\lambda}{\lambda+1}\right)^{N} \sqrt{\frac{-\theta_{0}}{\Theta^{+}(y, \gamma)-\theta_{0}}}} \\
& \times \sqrt{\frac{1}{\Psi_{99}\left[y, 1, \Theta^{+}(y, \gamma), \gamma\right]}} \exp \left\{N \Psi\left[y, 1, \Theta^{+}(y, \gamma), \gamma\right]\right\}
\end{aligned}
$$

since

$$
\begin{gathered}
\frac{\partial \Psi}{\partial z}\left(y, W_{+}, \Theta^{+}, z\right)=0 \quad \text { for } z=\gamma, \\
\frac{\partial^{2} \Psi}{\partial z^{2}}\left(y, W_{+}, \Theta^{+}, z\right)=\frac{\Theta^{+}}{\rho} \quad \text { for } z=\gamma, \\
W_{+}(\vartheta, \gamma)=1, \quad \eta_{w w}(1, \vartheta, \gamma)=-\frac{\rho}{\vartheta} .
\end{gathered}
$$

Therefore,

$$
\begin{aligned}
f(k \mid x) \sim & \frac{1}{\sqrt{2 \pi N}} \frac{1}{W_{+}\left(\Theta^{+}, z\right)} \sqrt{\frac{\Theta^{+}(y, \gamma)-\theta_{0}}{\Theta^{+}-\theta_{0}}} \sqrt{\frac{1}{\eta_{w w}\left(W_{+}, \Theta^{+}, z\right)}} \\
& \times \sqrt{\frac{\Psi_{\vartheta \vartheta}\left[y, 1, \Theta^{+}(y, \gamma), \gamma\right]}{\Psi_{9 \vartheta}\left(y, W_{+}, \Theta^{+}, z\right)}} \exp \left\{N \Psi\left(y, W_{+}, \Theta^{+}, z\right)-N \Psi\left[y, 1, \Theta^{+}(y, \gamma), \gamma\right]\right\},
\end{aligned}
$$


and expanding this for $z \rightarrow \gamma$ yields the Gaussian form

$$
\begin{aligned}
f(k \mid x)= & f(N \gamma+\sqrt{N} \Lambda \mid N y) \sim \frac{1}{\sqrt{2 \pi N}} \sqrt{\frac{-\Theta^{+}(y, \gamma)}{\rho}} \\
& \times \exp \left\{\frac{1}{2} \frac{\partial^{2} \Psi}{\partial z^{2}}\left[y, 1, \Theta^{+}(y, \gamma), \gamma\right](z-\gamma)^{2} N\right\} \\
= & \frac{1}{\sqrt{2 \pi N}} \sqrt{\frac{-\Theta^{+}(y, \gamma)}{\rho}} \exp \left\{\frac{1}{2} \frac{\Theta^{+}(y, \gamma)}{\rho} \Lambda^{2}\right\},
\end{aligned}
$$

with $\Lambda=\sqrt{N}(z-\gamma)$. This means that given a buffer size $x=N y, y>0$, the most likely number of active sources will be $c=N \gamma$, with a Gaussian spread whose variance is obtained by solving (4.72), with $z=\gamma$. This is also illustrated in Figure 10.7.

Next, we assume a small buffer size, with $x=O(1 / N)$. Again the main contribution to the sum (11.1) comes from $k \approx c$, but for $x=O(1 / N)$, we must use the Region I approximation $f_{k}(x) \sim N F_{l}^{(1)^{\prime}}(\chi)$. Thus,

$$
\begin{aligned}
f_{k}(x) \sim & \frac{\sqrt{N}}{\sqrt{2 \pi}} \sqrt{\frac{\rho}{\gamma \phi(1-\gamma)}}\left(\frac{\beta}{2 \gamma}\right)^{l-\alpha} \exp [N \Phi(\gamma)] \\
& \times \frac{1}{2 \pi i} \int_{\mathrm{Br}^{+}} e^{\chi S} \Gamma\left(\frac{\phi}{S}+1-\alpha\right) J_{l-\alpha+\phi / S}\left(\frac{\beta}{S}\right) \\
& \times \exp \left\{\frac{\phi}{S} \ln \left[\frac{\beta S}{2 \lambda \phi(1-\gamma)}\right]+\frac{\phi-\rho}{S}\right\}\left(\frac{\phi}{S}\right)^{\alpha} d S
\end{aligned}
$$

which we can write as a residue series

$$
\begin{aligned}
f_{k}(x) \sim & \frac{\sqrt{N}}{\sqrt{2 \pi}} \sqrt{\frac{\rho \phi}{\gamma(1-\gamma)}}\left(\frac{\beta}{2 \gamma}\right)^{l-\alpha} \exp [N \Phi(\gamma)] \\
& \times \sum_{j=0}^{\infty} \exp \left[-\frac{\phi}{j+1-\alpha} \chi+\frac{(j+1-\alpha)(\rho-\phi)}{\phi}\right] \\
& \times \frac{(j+1-\alpha)^{j-1}}{j !}\left[\frac{2 \lambda(1-\gamma)}{\beta}\right]^{j+1-\alpha} J_{l-j-1}\left[(\alpha-1-j) \frac{\beta}{\phi}\right],
\end{aligned}
$$

where we have used

$$
\operatorname{Res}\left[\Gamma\left(\frac{\phi}{S}+1-\alpha\right), S=\frac{\phi}{\alpha-1-j}\right]=\frac{(-1)^{j+1} \phi}{j !(j+1-\alpha)^{2}}, \quad j \geq 0 .
$$

Using the generating function [52]

$$
\sum_{j=-\infty}^{\infty} J_{j}(x) z^{j}=\exp \left[\frac{x}{2}\left(z-\frac{1}{z}\right)\right]
$$

in (11.11), we obtain

$$
\sum_{l=-\infty}^{\infty}\left(\frac{\beta}{2 \gamma}\right)^{l-(j+1)} J_{l-j-1}\left[(\alpha-1-j) \frac{\beta}{\phi}\right]=\exp \left[\frac{\rho}{\phi}(j+1-\alpha)\right],
$$


and thus

$$
\begin{aligned}
M_{1}(x) \sim & \frac{\sqrt{N}}{\sqrt{2 \pi}} \sqrt{\frac{\rho \phi}{\gamma(1-\gamma)}} \exp [N \Phi(\gamma)] \\
& \times \sum_{j=0}^{\infty} \exp \left[-\frac{\phi}{j+1-\alpha} \chi\right] \frac{(j+1-\alpha)^{j-1}}{j !} \exp \left[\frac{2 \rho-\phi}{\phi}(j+1-\alpha)\right]\left[\frac{\lambda(1-\gamma)}{\gamma}\right]^{j+1-\alpha} .
\end{aligned}
$$

It follows that

$$
\begin{aligned}
& f(k \mid x)=f\left(c+l-\alpha \mid \frac{\chi}{N}\right) \\
& \sim \frac{\left(\frac{\beta}{2 \gamma}\right)^{l-\alpha} \sum_{j=0}^{\infty} \exp \left[-\frac{\phi}{j+1-\alpha} \chi+\frac{(j+1-\alpha)(\rho-\phi)}{\phi}\right] \frac{(j+1-\alpha)^{j-1}}{j !}\left[\frac{2 \lambda(1-\gamma)}{\beta}\right]^{j+1-\alpha} J_{l-j-1}\left[(\alpha-1-j) \frac{\beta}{\phi}\right]}{\sum_{j=0}^{\infty} \exp \left[-\frac{\phi}{j+1-\alpha} \chi+\frac{2 \rho-\phi}{\phi}(j+1-\alpha)\right] \frac{(j+1-\alpha)^{j-1}}{j !}\left[\frac{\lambda(1-\gamma)}{\gamma}\right]^{j+1-\alpha}}
\end{aligned}
$$

which is a discrete distribution in $l$.

11.2. The buffer density conditioned on $k$. We first consider $\gamma<z \leq 1$. In this range, $F_{k}(0)=0$, and from (11.4), we have $M_{2}(k)=F_{k}(\infty)$. We have

$$
\frac{\partial \Psi}{\partial y}\left[y, W_{-}, \Theta, z\right]=\Theta(y, z)
$$

so that the maximum of the density occurs at $\Theta=0$, which corresponds to the transition curve

$$
y=Y_{0}(z)=\frac{z-\gamma}{\lambda+1}-\frac{\rho}{(\lambda+1)^{2}} \ln \left(\frac{z \lambda+z-\lambda}{\rho}\right), \quad \gamma<z<1 .
$$

Therefore, we will use the approximate solution $F^{(2)}(V, z)$ since Region II corresponds to $\Theta \approx 0$. From (10.3) we get, for $\gamma<z<1$,

$$
\begin{aligned}
f_{k}(x)= & \frac{1}{N} \frac{\partial F^{(2)}}{\partial V} \frac{\partial V}{\partial y} \sim \frac{1}{2 \pi N} \sqrt{\frac{1}{z(1-z)}} \exp [\Phi(z) N] \frac{1}{\sqrt{Y_{2}(z)}} \exp \left(-\frac{V^{2}}{2}\right) \\
Y_{2}(z)= & \frac{2 \zeta}{(\lambda+1)^{4}} \ln \left(\frac{z+z \lambda-\lambda}{\rho}\right) \\
& -\frac{z-\gamma}{(\lambda+1)(\lambda z+z-\lambda)^{2}}\left[\frac{2 \zeta \rho}{(\lambda+1)^{2}}+\frac{3 \zeta}{(\lambda+1)}(z-\gamma)+(\lambda-1)(z-\gamma)^{2}\right] .
\end{aligned}
$$

From (11.4), we obtain

$$
M_{2}(k)=F_{k}(\infty) \sim \frac{1}{\sqrt{2 \pi N}} \sqrt{\frac{1}{z(1-z)}} \exp [\Phi(z) N],
$$


and we conclude that

$$
f(x \mid k)=f\left[N Y_{0}(z)+\sqrt{N} \Omega \mid N z\right] \sim \frac{1}{\sqrt{2 \pi}} \frac{1}{\sqrt{Y_{2}(z) N}} \exp \left\{-\frac{1}{2} \frac{\Omega^{2}}{Y_{2}(z)}\right\},
$$

with $\Omega=\left[y-Y_{0}(z)\right] \sqrt{N}$. This shows that if the number of active sources exceeds the service rate, the buffer content is likely to be large, approximately $N Y_{0}(z)$ and with a Gaussian spread proportional to $Y_{2}(z)$ (see also Figure 10.2). While this calculation assumed that $\gamma<z<1$, we can show that the result remains valid for $z \rightarrow 1$.

Next, we consider the range $0 \leq z<\gamma$. We use the asymptotic approximation $F_{k}(x)-$ $F_{k}(\infty) \sim G_{2}(y, z)$ or

$$
\begin{aligned}
f_{k}(x) \sim & \frac{1}{2 \pi N}\left(\frac{\lambda}{\lambda+1}\right)^{N} \frac{1}{W_{+}\left(\Theta^{+}, z\right)} \sqrt{\frac{-\theta_{0}}{\Theta^{+}-\theta_{0}}} \sqrt{\frac{1}{\eta_{w w}\left(W_{+}, \Theta^{+}, z\right)}} \\
& \times \sqrt{\frac{1}{\Psi_{99}\left(y, W_{+}, \Theta^{+}, z\right)}} \exp \left[N \Psi\left(y, W_{+}, \Theta^{+}, z\right)\right] .
\end{aligned}
$$

From (11.4), we have

$$
M_{2}(k)=F_{k}(\infty)-F_{k}(0) \sim-G_{2}(0, z) .
$$

Hence,

$$
\begin{aligned}
f(x \mid k) \sim & -\Theta^{+}(0, z) \frac{W_{+}\left[\Theta^{+}(0, z), z\right]}{W_{+}\left(\Theta^{+}, z\right)} \sqrt{\frac{\Theta^{+}(0, z)-\theta_{0}}{\Theta^{+}-\theta_{0}}} \sqrt{\frac{\Psi_{\vartheta \vartheta}\left[0, W_{+}, \Theta^{+}(0, z), z\right]}{\Psi_{\vartheta \vartheta}\left(y, W_{+}, \Theta^{+}, z\right)}} \\
& \times \sqrt{\frac{\eta_{w w}\left[W_{+}, \Theta^{+}(0, z), z\right]}{\eta_{w w}\left(W_{+}, \Theta^{+}, z\right)}} \exp \left\{N \Psi\left[y, W_{+}, \Theta^{+}, z\right]-N \Psi\left[0, W_{+}, \Theta^{+}(0, z), z\right]\right\},
\end{aligned}
$$

and as $y \rightarrow 0$,

$$
\begin{aligned}
f(x \mid k) & =f(x \mid N z) \sim-\Theta^{+}(0, z) \exp \left[\left\{N \frac{\partial \Psi}{\partial y}\left[0, W_{+}, \Theta^{+}(0, z), z\right] y\right\}\right] \\
& =-\Theta^{+}(0, z) \exp \left[\Theta^{+}(0, z) x\right] .
\end{aligned}
$$

This shows that if the number of on sources is less than the service rate $c$ and the buffer is not empty, the conditional density is approximately exponential in $x$, with mean $1 /$ $-\Theta^{+}(0, z)$. This exponential shape is also illustrated in Figure 10.3.

Finally, we consider the case $k \approx c$. We will again use the approximation in Region $\mathrm{I}$, and from (11.11), we get

$$
\begin{aligned}
M_{2}(k) \sim & \frac{1}{\sqrt{2 \pi N}} \sqrt{\frac{\rho}{\gamma \phi(1-\gamma)}}\left(\frac{\beta}{2 \gamma}\right)^{l-\alpha} \exp [N \Phi(\gamma)] \sum_{j=0}^{\infty} \exp \left[\frac{(j+1-\alpha)(\rho-\phi)}{\phi}\right] \\
& \times \frac{(j+1-\alpha)^{j}}{j !}\left[\frac{2 \lambda(1-\gamma)}{\beta}\right]^{j+1-\alpha} J_{l-j-1}\left[(\alpha-1-j) \frac{\beta}{\phi}\right] .
\end{aligned}
$$


Therefore,

$$
\begin{aligned}
& f(x \mid k)=f\left(\frac{\chi}{N} \mid c+l-\alpha\right) \\
& \sim \frac{\phi N \sum_{j=0}^{\infty} \exp \left[-\frac{\phi}{j+1-\alpha} \chi+\frac{(j+1-\alpha)(\rho-\phi)}{\phi}\right] \frac{(j+1-\alpha)^{j-1}}{j !}\left[\frac{2 \lambda(1-\gamma)}{\beta}\right]^{j+1-\alpha} J_{l-j-1}\left[(\alpha-1-j) \frac{\beta}{\phi}\right]}{\sum_{j=0}^{\infty} \exp \left[\frac{(j+1-\alpha)(\rho-\phi)}{\phi}\right] \frac{(j+1-\alpha)^{j}}{j !}\left[\frac{2 \lambda(1-\gamma)}{\beta}\right]^{j+1-\alpha} J_{l-j-1}\left[(\alpha-1-j) \frac{\beta}{\phi}\right]}
\end{aligned}
$$

which is a density in $\chi$, consisting of an infinite mixture of exponentials.

\section{Acknowledgments}

The work of C. Knessl was partially supported by NSF Grants DMS 99-71656, DMS 0202815 and NSA Grant MDA 904-03-1-0036. The work of D. Dominici was supported in part by NSF Grant 99-73231, provided by Professor Floyd Hanson. We wish to thank him for his generous sponsorship.

\section{References}

[1] M. Abramowitz and I. A. Stegun (eds.), Handbook of Mathematical Functions with Formulas, Graphs, and Mathematical Tables, Dover Publications, New York, 1992, reprint of the 1972.

[2] I. Adan and J. Resing, Simple analysis of a fluid queue driven by an $M / M / 1$ queue, Queueing Syst. Theory Appl. 22 (1996), no. 1-2, 171-174.

[3] D. Anick, D. Mitra, and M. M. Sondhi, Stochastic theory of a data-handling system with multiple sources, Bell System Tech. J. 61 (1982), no. 8, 1871-1894.

[4] N. Barbot and B. Sericola, Stationary solution to the fluid queue fed by an M/M/1 queue, J. Appl. Probab. 39 (2002), no. 2, 359-369.

[5] C. M. Bender and S. A. Orszag, Advanced Mathematical Methods for Scientists and Engineers, International Series in Pure and Applied Mathematics, McGraw-Hill Book, New York, 1978.

[6] N. Bleistein and R. A. Handelsman, Asymptotic Expansions of Integrals, Dover Publications, New York, 1986.

[7] W. W. Chu and L. C. Liang, Buffer behavior for mixed input traffic and single constant output rate, IEEE Trans. Comm. 20 (1972), no. 2, 230-235.

[8] E. G. Coffman, B. M. Igelnik, and Y. A. Kogan, Controlled stochastic model of a communication system with multiple sources, IEEE Trans. Inform. Theory 37 (1991), no. 5, 1379-1387.

[9] C. Courcoubetis and R. Weber, Buffer overflow asymptotics for a buffer handling many traffic sources, J. Appl. Probab. 33 (1996), no. 3, 886-903.

[10] J. N. Daigle and J. D. Langford, Models for analysis of packet voice communications systems, IEEE J. on Selected Areas in Commun. 4 (1986), no. 6, 847-855.

[11] N. G. Duffield, Conditioned asymptotics for tail probabilities in large multiplexers, Perform. Eval. 31 (1998), no. 3-4, 281-300.

[12] V. Dumas and A. Simonian, Asymptotic bounds for the fluid queue fed by sub-exponential On/Off sources, Adv. in Appl. Probab. 32 (2000), no. 1, 244-255.

[13] B. V. Gnedenko, The Theory of Probability, translated from the 4th Russian edition by B. D. Seckler, Chelsea Publishing, New York, 1967.

[14] I. S. Gradshteyn and I. M. Ryzhik, Table of Integrals, Series, and Products, Academic Press, California, 2000, translated from Russian. 
[15] O. Hashida and M. Fujiki, Queueing models for buffer memory in store-and-forward systems, Proceedings of the 7th International Teletraffic Congress, Stockholm, 1973, pp. 323-327.

[16] C. Knessl and J. A. Morrison, Heavy-traffic analysis of a data-handling system with many sources, SIAM J. Appl. Math. 51 (1991), no. 1, 187-213.

[17] K. Knopp, Theory of Functions. I. Elements of the General Theory of Analytic Functions, Dover Publications, New York, 1945.

[18] H. Kobayashi and Q. Ren, A mathematical theory for transient analysis of communication networks, IEICE Transactions on Communications E75-B (1992), no. 12, 1266-1276.

[19] - Transient solutions for the buffer behavior in statistical multiplexing, Perform. Eval. 23 (1995), no. 1, 65-87.

[20] V. Konovalov, Fluid queue driven by a GI $\mid$ GI $\mid 1$ queue, J. Math. Sci. (New York) 91 (1998), no. 3, 2917-2930.

[21] L. Kosten, Stochastic theory of a multi-entry buffer. I, Delft Progr. Rep. 1 (1974), no. 1, 10-18.

[22] - Stochastic theory of a multi-entry buffer. II, Delft Progr. Rep. 1 (1974), no. 2, 44-50.

[23] Stochastic theory of data-handling systems with groups of multiple sources, Proceedings of the 2nd International Symposium on the Performance of Computer-Communication Systems (H. Rudin and W. Bux, eds.), Elsevier Science Publishers, Amsterdam, 1984, pp. 321-331.

[24] Liquid models for a type of information buffer problems, Delft Progr. Rep. 11 (1986), no. 2, 71-86.

[25] D. P. Kroese and W. R. W. Scheinhardt, Joint distributions for interacting fluid queues, Queueing Syst. Theory Appl. 37 (2001), no. 1-3, 99-139.

[26] V. G. Kulkarni, Fluid models for single buffer systems, Frontiers in Queueing, Probability and Stochastics Series, CRC Press, Florida, 1997, pp. 321-338.

[27] V. G. Kulkarni and E. Tzenova, Mean first passage times in fluid queues, Oper. Res. Lett. 30 (2002), no. 5, 308-318.

[28] S.-Q. Li, A new performance measurement for voice transmission in burst and packet switching, IEEE Trans. Comm. 35 (1987), no. 10, 1083-1094.

[29] Study of information loss in packet voice systems, IEEE Trans. Comm. 37 (1989), no. 11, $1192-1202$.

[30] Y. Liu and W. Gong, Perturbation analysis for stochastic fluid queueing systems, Discrete Event Dyn. Syst. 12 (2002), no. 4, 391-416.

[31] M. Mandjes, Overflow asymptotics for large communications systems with general Markov fluid sources, Probab. Engrg. Inform. Sci. 10 (1996), no. 4, 501-518.

[32] M. Mandjes and A. Ridder, Finding the conjugate of Markov fluid processes, Probab. Engrg. Inform. Sci. 9 (1995), no. 2, 297-315.

[33] D. Mitra, Stochastic theory of a fluid model of producers and consumers coupled by a buffer, Adv. in Appl. Probab. 20 (1988), no. 3, 646-676.

[34] J. A. Morrison, Asymptotic analysis of a data-handling system with many sources, SIAM J. Appl. Math. 49 (1989), no. 2, 617-637.

[35] A. Narayanan and V. G. Kulkarni, First passage times in fluid models with an application to two priority fluid systems, Proceedings of the 2 nd International Computer Performance and Dependability Symposium (IPDS '96), Institute of Electrical and Electronics Engineers (IEEE), New York, 1996, pp. 166-175.

[36] F. W. J. Olver, Asymptotics and Special Functions, AKP Classics, AK Peters, Massachusetts, 1997.

[37] A. Pacheco and N. U. Prabhu, A Markovian storage model, Ann. Appl. Probab. 6 (1996), no. 1, 76-91.

[38] P. R. Parthasarathy, K. V. Vijayashree, and R. B. Lenin, An M/M/1 driven fluid queue-continued fraction approach, Queueing Syst. Theory Appl. 42 (2002), no. 2, 189-199. 
[39] S. Resnick and G. Samorodnitsky, Steady-state distribution of the buffer content for $M / G / \infty$ input fluid queues, Bernoulli 7 (2001), no. 2, 191-210.

[40] H. Rudin, Buffered packet switching: a queue with clustered arrivals, Proceedings of the International Switching Symposium, MIT Press, Massachusetts, 1972.

[41] W. R. W. Scheinhardt, Markov-modulated and feedback fluid queues, Ph.D. thesis, Faculty of Mathematical Sciences, University of Twente, Enschede, 1998.

[42] B. Sericola, Transient analysis of stochastic fluid models, Perform. Eval. 32 (1998), no. 4, 245263.

[43] _ A finite buffer fluid queue driven by a Markovian queue, Queueing Syst. Theory Appl. 38 (2001), no. 2, 213-220.

[44] B. Sericola and B. Tuffin, A fluid queue driven by a Markovian queue, Queueing Syst. Theory Appl. 31 (1999), no. 3-4, 253-264.

[45] B. A. Sevast'yanov, Influence of storage bin capacity on the average standstill time of a production line, Theory Probab. Appl. 7 (1962), 429-438.

[46] A. Shwartz and A. Weiss, Large Deviations for Performance Analysis, Stochastic Modeling Series, Chapman \& Hall, London, 1995.

[47] G. Szegő, Orthogonal Polynomials, American Mathematical Society, Colloquium Publications, vol. 23, American Mathematical Society, Rhode Island, 1975.

[48] H. Takada, Markov modulated fluid queues with batch fluid arrivals, J. Oper. Res. Soc. Japan 44 (2001), no. 4, 344-365.

[49] T. Tanaka, O. Hashida, and Y. Takahashi, Transient analysis of fluid model for ATM statistical multiplexer, Perform. Eval. 23 (1995), no. 2, 145-162.

[50] R. C. F. Tucker, Accurate method for analysis of a packet-speech multiplexer with limited delay, IEEE Trans. Comm. 36 (1988), no. 4, 479-483.

[51] E. A. van Doorn and W. R. W. Scheinhardt, Analysis of birth-death fluid queues, Proceedings of the Applied Mathematics Workshop, vol. 5, Korea Advanced Institute of Science and Technology, Taejon, 1996, pp. 13-29.

[52] G. N. Watson, A Treatise on the Theory of Bessel Functions, Cambridge Mathematical Library, Cambridge University Press, Cambridge, 1995.

[53] A. Weiss, A new technique for analyzing large traffic systems, Adv. in Appl. Probab. 18 (1986), no. 2, 506-532.

[54] E. T. Whittaker and G. N. Watson, A Course of Modern Analysis, Cambridge Mathematical Library, Cambridge University Press, Cambridge, 1996.

[55] J. Wijngaard, The effect of interstage buffer storage on the output of two unreliable production units in series with different production rates, AIIE Trans. 11 (1979), no. 1, 42-47.

Diego Dominici: Department of Mathematics, Statistics and Computer Science, University of Illinois at Chicago, M/C 249, 851 South Morgan Street, Chicago, IL 60607-7045, USA

E-mail address: ddomin1@uic.edu

Charles Knessl: Department of Mathematics, Statistics and Computer Science, University of Illinois at Chicago, M/C 249, 851 South Morgan Street, Chicago, IL 60607-7045, USA

E-mail address: knessl@uic.edu 


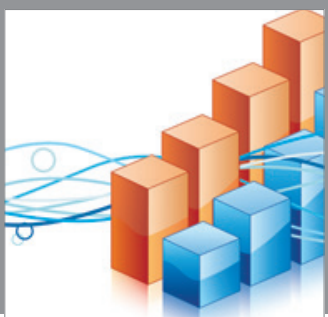

Advances in

Operations Research

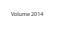

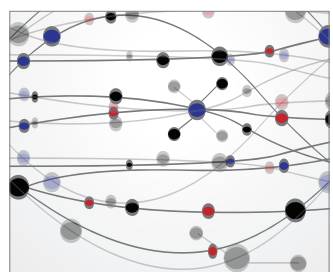

\section{The Scientific} World Journal
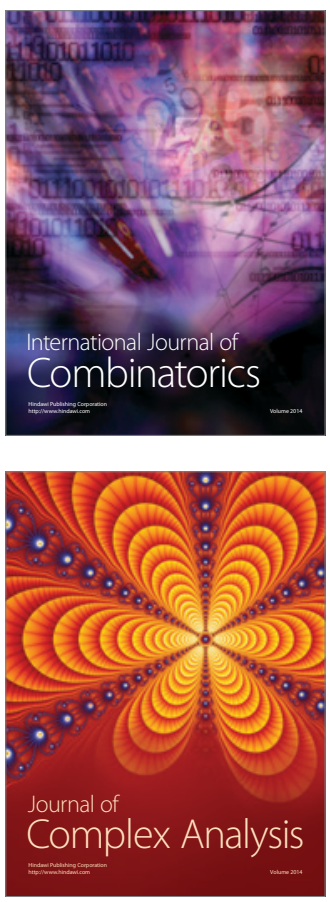

International Journal of

Mathematics and

Mathematical

Sciences
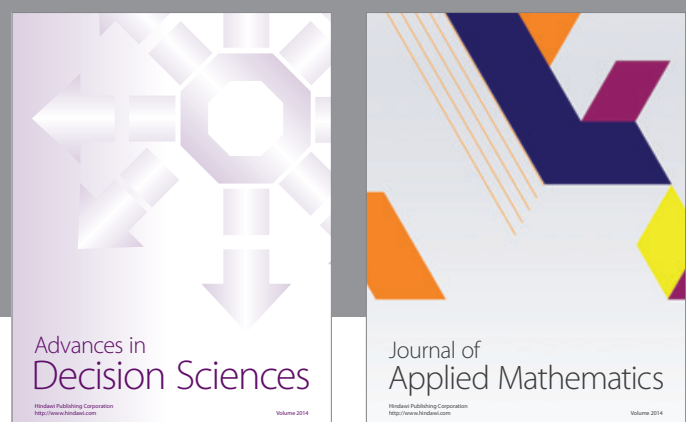

Journal of

Applied Mathematics
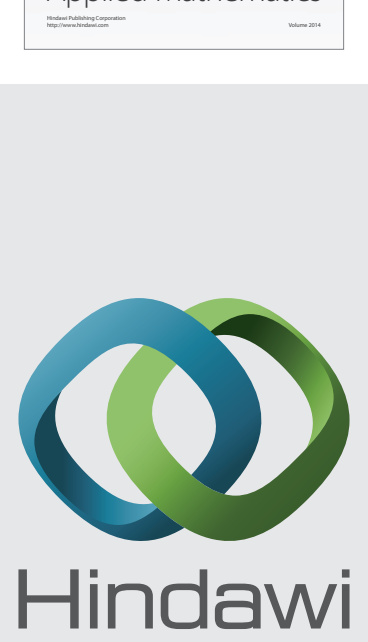

Submit your manuscripts at http://www.hindawi.com
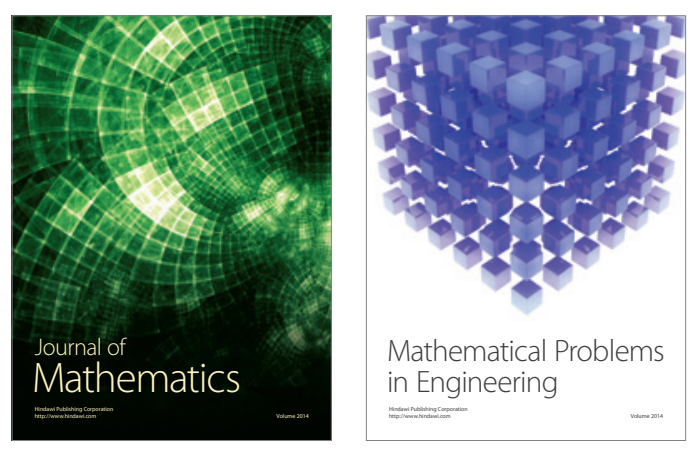

Mathematical Problems in Engineering
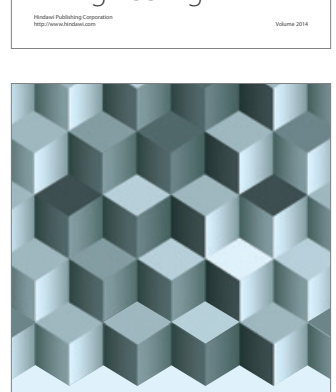

Journal of

Function Spaces
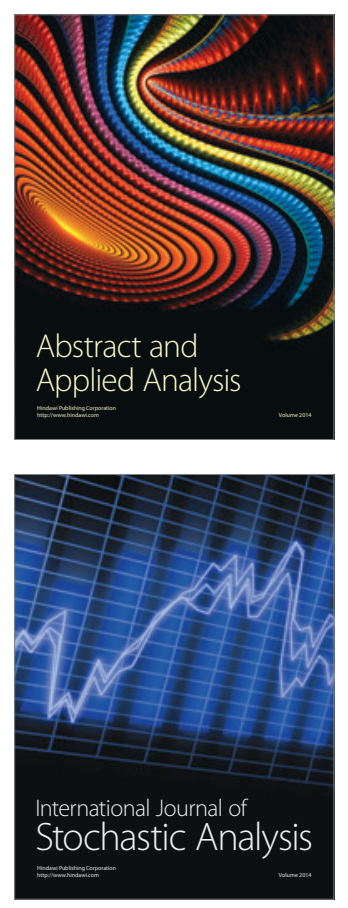

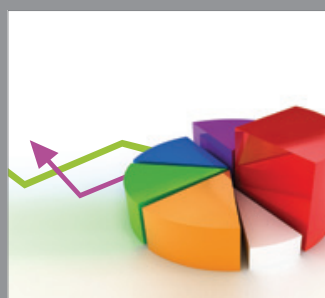

ournal of

Probability and Statistics

Promensencen
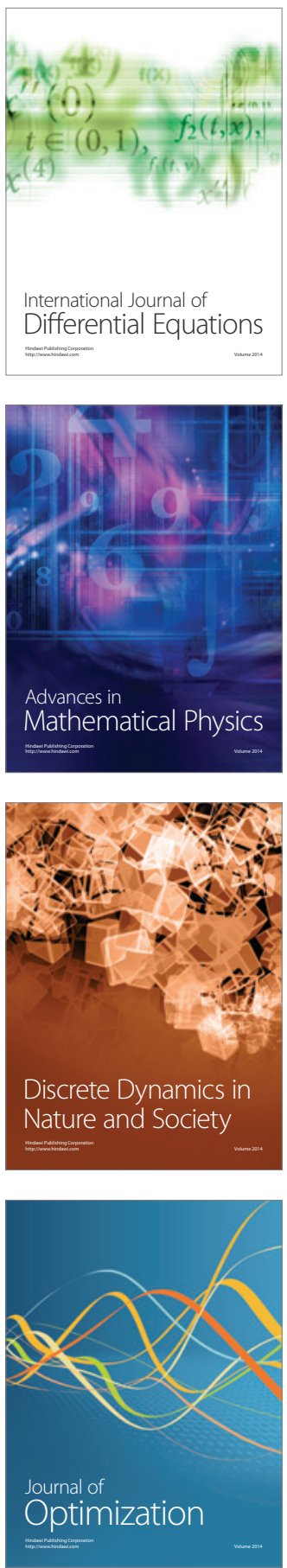